\title{
Strong Anisotropic MHD Turbulence with Cross Helicity
}

\author{
Benjamin D. G. Chandran \\ benjamin. chandran@unh.edu \\ Space Science Center and Department of Physics, University of New Hampshire
}

\begin{abstract}
This paper proposes a new phenomenology for strong incompressible MHD turbulence with nonzero cross helicity. This phenomenology is then developed into a quantitative Fokker-Planck model that describes the time evolution of the anisotropic power spectra of the fluctuations propagating parallel and anti-parallel to the background magnetic field $B_{0}$. It is found that in steady state the power spectra of the magnetic field and total energy are steeper than $k_{\perp}^{-5 / 3}$ and become increasingly steep as $C / \mathcal{E}$ increases, where $C=\int d^{3} x v \cdot B$ is the cross helicity, $\mathcal{E}$ is the fluctuation energy, and $k_{\perp}$ is the wavevector component perpendicular to $B_{0}$. Increasing $C$ with fixed $\mathcal{E}$ increases the time required for energy to cascade to smaller scales, reduces the cascade power, and increases the anisotropy of the small-scale fluctuations. The implications of these results for the solar wind and solar corona are discussed in some detail.
\end{abstract}

Subject headings: turbulence — magnetic fields — magnetohydrodynamics — solar wind - solar corona — solar flares

\section{Introduction}

Much of our current understanding of incompressible magnetohydrodynamic (MHD) turbulence has its roots in the pioneering work of Iroshnikov (1963) and Kraichnan (1965). These studies emphasized the important fact that Alfvén waves travelling in the same direction along a background magnetic field do not interact with one another and explained how one can think of the cascade of energy to small scales as resulting from collisions between oppositely directed Alfvén wave packets. They also argued that in the absence of a mean magnetic field, the magnetic field of the energy-containing eddies at scale $L$ affects fluctuations on scales $\ll L$ much in the same way as would a truly uniform mean magnetic field. 
Another foundation of our current understanding is the finding that MHD turbulence is inherently anisotropic. Montgomery \& Turner (1981) and Shebalin, Matthaeus, \& Montgomery (1983) showed that a strong uniform mean magnetic field $B_{0}$ inhibits the cascade of energy to small scales measured in the direction parallel to $B_{0}$. This early work was substantially elaborated upon by Higdon (1984), Goldreich \& Sridhar (1995, 1997), Montgomery \& Matthaeus (1995), Ng \& Bhattacharjee (1996, 1997), Galtier et al (2000), Cho \& Lazarian (2003), Oughton et al (2006), and many others. For example, Cho \& Vishniac (2000) used numerical simulations to show that when the fluctuating magnetic field $\delta B$ is $\gtrsim B_{0}$ the small-scale turbulent eddies become elongated along the local magnetic field direction. Goldreich \& Sridhar (1995) introduced the important and influential idea of "critical balance," which holds that at each scale the linear wave period for the bulk of the fluctuation energy is comparable to the time for the fluctuation energy to cascade to smaller scales. Goldreich \& Sridhar (1995, 1997), Maron \& Goldreich (2001), and Lithwick \& Goldreich (2001) clarified a number of important physical processes in anisotropic MHD turbulence and used the concept of critical balance to determine the ratio of the dimensions of turbulent eddies in the directions parallel and perpendicular to the local magnetic field.

Over the last several years, research on MHD turbulence has been proceeding along several different lines. For example, one group of studies has attempted to determine the power spectrum, intermittency, and anisotropy of strong incompressible MHD turbulence using direct numerical simulations. (See, e.g., Cho \& Vishniac 2000, Müller \& Biskamp 2000, Maron \& Goldreich 2001, Cho et al 2002, Haugen et al 2004, Muller \& Grappin 2005, Mininni \& Pouquet 2007, Perez \& Boldyrev 2008). Another series of papers has explored the properties of anisotropic turbulence in weakly collisional magnetized plasmas using gyrokinetics, a low-frequency expansion of the Vlasov equation that averages over the gyromotion of the particles. (Howes et al 2006, 2007a, 2007b; Schekochihin et al 2007). These authors investigated the transition between the Alfvénwave cascade and a kinetic-Alfvén-wave cascade at length scales of order the proton gyroradius $\rho_{i}$, as well as the physics of energy dissipation and entropy production in the low-collisionality regime. Turbulence at scales $\lesssim \rho_{i}$ has also been examined both numerically and analytically within the framework of fluid models, in particular Hall MHD and electron MHD. (Biskamp, Schwarz, \& Drake 1996, Biskamp et al 1999, Matthaeus et al 2003; Galtier \& Bhattacharjee 2003, 2005; Cho \& Lazarian 2004; Brodin et al 2006, Shukla et al 2006). Another group of studies has investigated the power spectrum, intermittency, and decay time of compressible MHD turbulence. (Oughton et al 1995, Stone et al 1998, Lithwick \& Goldreich 2001, Boldyrev et al 2002, Padoan et al 2004, Elmegreen \& Scalo 2004). Additional work by Kuznetsov (2001), Cho \& Lazarian (2002, 2003), Chandran (2005), and Luo \& Melrose (2006) has begun to address the way in which Alfvén waves, fast magnetosonic waves, and slow magnetosonic waves interact in compressible weak MHD turbulence. Another recent development is the finding that strong incompressible MHD turbulence leads to alternating patches of alignment and anti-alignment between the veloc- 
ity and magnetic-field fluctuations. (Boldyrev 2005, 2006; Beresnyak \& Lazarian 2006; Mason, Cattaneo, \& Boldyrev 2006; Matthaeus et al 2007) These studies examined how the degree of local alignment (and anti-alignment) depends upon length scale, as well as the effects of alignment upon the energy cascade time and the power spectrum of the turbulence.

The topic addressed in this paper is the role of cross helicity in incompressible MHD turbulence. The cross helicity is defined as

$$
C=\int d^{3} x v \cdot B
$$

where $v$ is the velocity and $B$ is the magnetic field. The cross helicity is conserved in the absence of dissipation and can be thought of as a measure of the linkages between lines of vorticity and magnetic field lines, both of which are frozen to the fluid flow in the absence of dissipation (Moffatt 1978). In the presence of a background magnetic field, $B_{0}=B_{0} \hat{z}$, the cross helicity is also a measure of the difference between the energy of fluctuations travelling in the $-z$ and $+z$ directions. Dobrowolny, Mangeney, \& Veltri (1980) showed that MHD turbulence with cross helicity decays to a maximally aligned state, with $\delta v= \pm \delta B / \sqrt{4 \pi \rho}$, where $\delta v$ and $\delta B$ are the fluctuating velocity and magnetic field and $\rho$ is the mass density. Different decay rates for the energy and cross helicity were also demonstrated by Matthaeus \& Montgomery (1980). In another early study, Grappin, Pouquet, \& Léorat (1983) used a statistical closure, the eddy-damped quasi-normal Markovian (EDQNM) approximation, to study strong 3D incompressible MHD turbulence with cross helicity, assuming isotropic power spectra. They found that when $C \neq 0$, the total energy spectrum is steeper than the isotropic Iroshnikov-Kraichnan $k^{-3 / 2}$ spectrum. Pouquet et al (1988) found a similar result in direct numerical simulations of 2D incompressible MHD turbulence. More recently, Lithwick, Goldreich, \& Sridhar (2007) and Beresnyak \& Lazarian (2007) addressed the role of cross helicity in strong MHD turbulence taking into account the effects of anisotropy.

This paper presents a new phenomenology for strong, anisotropic, incompressible MHD turbulence with nonzero cross helicity, and is organized as follows. Section 2 presents some relevant theoretical background. Section 3 introduces the new phenomenology as well as two nonlinear advection-diffusion equations that model the time evolution of the power spectra. Analytic and numerical solutions to this equation in the weak-turbulence and strong-turbulence regimes are presented in Sections 4 and 5. Section 5 also presents a simple phenomenological derivation of the power spectra and anisotropy in strong MHD turbulence. Section 6 presents a numerical solution to the advection-diffusion equation that shows the smooth transition between the weak and strong turbulence regimes. Section 7 addresses the case in which the parallel correlation lengths of waves propagating in opposite directions along the background magnetic field are unequal at the outer scale. In Section 8 , the proposed phenomenology is applied to turbulence in the solar wind and solar corona, and in Section 9 the results of this work are compared to the recent studies of Lithwick, Goldreich, \& Sridhar (2007) and Beresnyak \& Lazarian (2007). 


\section{Energy Cascade from Wave-Packet Collisions}

The equations of ideal incompressible MHD can be written

$$
\frac{\partial w^{ \pm}}{\partial t}+\left(w^{\mp} \mp v_{\mathrm{A}} \hat{z}\right) \cdot \nabla w^{ \pm}=-\nabla \Pi
$$

where $w^{ \pm}=v \pm(\delta B / \sqrt{4 \pi \rho})$ are the Elsasser variables, $v$ is the fluid velocity, $\delta B$ is the magnetic field fluctuation, $\rho$ is the mass density, which is taken to be uniform and constant, $v_{A}=B_{0} / \sqrt{4 \pi \rho}$ is the Alfvén speed, $B_{0}=B_{0} \hat{z}$ is the mean magnetic field, and $\Pi=\left(p+B^{2} / 8 \pi\right) / \rho$, which is determined by the incompressibility condition, $\nabla \cdot w^{ \pm}=0$. Throughout this paper it is assumed that $\delta B \lesssim B_{0}$ and $w^{ \pm} \lesssim v_{A}$.

In the limit of small-amplitude fluctuations $\left(w^{ \pm} \ll v_{A}\right)$, the nonlinear term $w^{\mp} \cdot \nabla w^{ \pm}$in equation (2) can be neglected to a first approximation, and the curl of equation (2) becomes

$$
\left(\frac{\partial}{\partial t} \mp v_{A} \frac{\partial}{\partial z}\right) \nabla \times w^{ \pm}=0
$$

which is solved by setting $\nabla \times w^{ \pm}$equal to an arbitrary function of $z \pm v_{A} t$. Thus, $w^{ \pm}$represents fluctuations with $v= \pm b$ that propagate in $\mp z$ direction at speed $v_{A}$ in the absence of nonlinear interactions. In the absence of an average velocity, the cross helicity defined in equation (1) can be rewritten as

$$
C=\frac{\sqrt{\pi \rho}}{2} \int d^{3} x\left[\left(w^{+}\right)^{2}-\left(w^{-}\right)^{2}\right] .
$$

The cross helicity is thus proportional to the difference in energy between fluctuations propagating in the $-z$ and $+z$ directions.

Equation (2) shows that the nonlinear term is nonzero only at those locations where both $w^{+}$ and $w^{-}$are nonzero. Nonlinear interactions can thus be thought of as collisions between oppositely directed wave packets (Kraichnan 1965). When both $w^{+}$and $w^{-}$are nonzero, equation (2) indicates that the $w^{ \pm}$fluctuations are advected not at the uniform velocity $\mp v_{A} \hat{z}$, but rather at the non-uniform velocity $\mp v_{A} \hat{z}+w^{\mp}$. Maron \& Goldreich (2001) elaborated upon this idea by showing that to lowest order in fluctuation amplitude, if one neglects the pressure term, then $w^{+}$ wave packets are advected along the hypothetical magnetic field lines corresponding to the sum of $B_{0}$ and the part of $\delta B$ arising from the $w^{-}$fluctuations. This result can be used to construct a geometrical picture for how wave-packet collisions cause energy to cascade to smaller scales, as depicted in Figure 1. In this figure, two oppositely directed wave packets of dimension $\sim \lambda_{\perp}$ in the plane perpendicular to $B_{0}$ and length $\lambda_{\|}$along $B_{0}$ pass through one another and get sheared. Collisions between wavepackets of similar $\lambda_{\perp}$ are usually the dominant mechanism for transferring energy from large scales to small scales. The duration of the collision illustrated in the figure is

approximately $\Delta t \sim \lambda_{\|} / v_{A}$. The fluctuating velocity and magnetic field are taken to be in the plane 
perpendicular to $B_{0}$, as is the case for linear shear Alfvén waves. The magnitude of the nonlinear term in equation (2) is then $\sim w_{\lambda_{\perp}}^{+} w_{\lambda_{\perp}}^{-} / \lambda_{\perp}$, where $w_{\lambda_{\perp}}^{ \pm}$is the rms amplitude of the $w^{ \pm}$wave packet. The fractional change in the $v$ and $b$ fields of the $w^{\mp}$ wave packet induced by the collision is then roughly

$$
\left(\frac{w_{\lambda_{\perp}}^{+} w_{\lambda_{\perp}}^{-}}{\lambda_{\perp}}\right) \times\left(\frac{\Delta t}{w_{\lambda_{\perp}}^{\mp}}\right)=\frac{w_{\lambda_{\perp}}^{ \pm} \lambda_{\|}}{v_{A} \lambda_{\perp}} .
$$

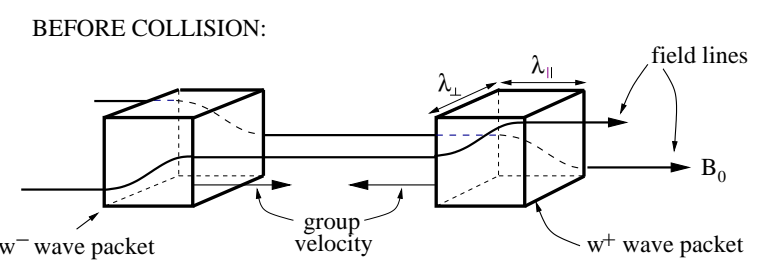

AFTER COLLISION:

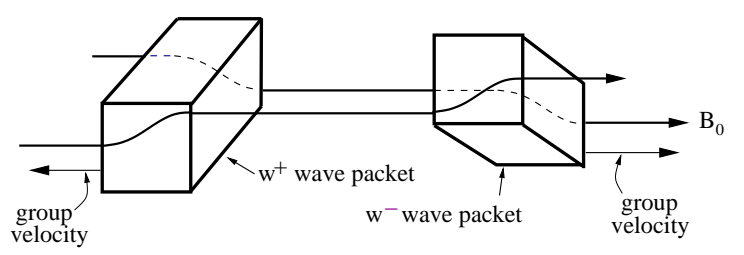

Fig. 1. - When two wave packets collide, each wave packet follows the field lines of the other wave packet and gets sheared.

If this fractional change is $\ll 1$ for both $w^{+}$and $w^{-}$fluctuations then neither wave packet is altered significantly by a single collision, and the turbulence is weak. Wave packets travel a distance $\gg \lambda_{\|}$before being significantly distorted, and the fluctuations can thus be viewed as linear waves that are only weakly perturbed by nonlinear interactions with other waves. In the wave-packet collision depicted in Figure 1, the right-hand side of the $w^{-}$wave packet is altered by the collision in almost the same way as the left-hand side, since both sides encounter essentially the same $w^{+}$wave packet, since the $w^{+}$packet is changed only slightly during the collision. Changes to the profile of a wave packet along the magnetic field are thus weaker than changes in the profile of a wave packet in the plane perpendicular to $B_{0}$ (Shebalin et al 1983, Ng \& Bhattacharjee 1997, Goldreich \& Sridhar 1997, Bhattacharjee \& Ng 2001, Perez \& Boldyrev 2008). As a result, in the weak-turbulence limit, the cascade of energy to small $\lambda_{\|}$is much less efficient than the cascade of energy to small $\lambda_{\perp}$ (Galtier et al 2000).

On the other hand, if the fractional change in equation (5) is of order unity then a $w^{\mp}$ wave packet is distorted substantially during a single collision, and the turbulence is said to be "strong." In the case that the fractional change in equation (5) is $\sim 1$ for one fluctuation type, (e.g., $w^{-}$) but $\ll 1$ for the other $\left(w^{+}\right)$, the turbulence is still referred to as strong. It should be noted that strong turbulence can arise when $w_{\lambda_{\perp}}^{ \pm} \ll v_{A}$, provided that $\lambda_{\perp} \ll \lambda_{\|}$. In strong turbulence energy cascades 
to smaller $\lambda_{\|}$to a greater extent than in weak turbulence, but the primary direction of energy flow in $k$-space is still to larger $k_{\perp}$, as discussed in the next section.

\section{Anisotropic MHD Turbulence with Cross Helicity}

In order to develop an analytical model, it is convenient to work in terms of the Fourier transforms of the fluctuating $w^{ \pm}$fields, given by

$$
\tilde{w}^{ \pm}(k)=\frac{1}{(2 \pi)^{3}} \int d^{3} x w(x) e^{-i k \cdot x} .
$$

The three-dimensional power spectrum $A^{ \pm}(k)$ is defined by the equation

$$
\left\langle\tilde{w}^{ \pm}(k) \cdot \tilde{w}^{ \pm}\left(k_{1}\right)\right\rangle=A^{ \pm}(k) \delta\left(k+k_{1}\right),
$$

where $\langle\ldots\rangle$ denotes an ensemble average. Cylindrical symmetry about $B_{0}$ is assumed, so that $A^{ \pm}(k)=A^{ \pm}\left(k_{\perp}, k_{\|}\right)$, where $k_{\perp}$ and $k_{\|}$are the components of $k$ perpendicular and parallel to $B_{0}$. The mean-square velocity associated with $w^{ \pm}$fluctuations is then

$$
\left(\delta v^{ \pm}\right)^{2}=\frac{1}{4} \int d^{3} k A^{ \pm}\left(k_{\perp}, k_{\|}\right)
$$

It is assumed that at each value of $k_{\perp}$ there is a parallel wave number $\bar{k}_{\|}^{ \pm}\left(k_{\perp}\right)$ such that (1) the bulk of the $w^{ \pm}$fluctuation energy is at $\left|k_{\|}\right|<\bar{k}_{\|}^{ \pm}\left(k_{\perp}\right)$ and (2) $A^{ \pm}\left(k_{\perp}, k_{\|}\right)$depends only weakly on $k_{\|}$for $\left|k_{\|}\right|<\bar{k}_{\|}^{ \pm}\left(k_{\perp}\right)$. A $w^{ \pm}$wavepacket at perpendicular scale $k_{\perp}^{-1}$ then has a correlation length in the direction of the mean field of $\sim\left(\bar{k}_{\|}^{ \pm}\right)^{-1}$. The rms amplitude of the fluctuating Elsasser fields at a perpendicular scale $k_{\perp}^{-1}$, denoted $w_{k_{\perp}}^{ \pm}$, is given by

$$
\left(w_{k_{\perp}}^{ \pm}\right)^{2} \sim A^{ \pm}\left(k_{\perp}, 0\right) k_{\perp}^{2} \bar{k}_{\|}^{ \pm}
$$

As described in the section 2 , when a $w^{\mp}$ wave packet at scale $k_{\perp}^{-1}$ collides with a $w^{ \pm}$wave packet at scale $k_{\perp}^{-1}$, the fractional change in the $w^{\mp}$ packet resulting from the collision is approximately

$$
\chi_{k_{\perp}}^{ \pm}=\frac{k_{\perp} w_{k_{\perp}}^{ \pm}}{\bar{k}_{\|}^{ \pm} v_{A}} .
$$

The wave number $k_{c}^{ \pm}$is defined to be the value of $\bar{k}_{\|}^{ \pm}$for which $\chi_{k_{\perp}}^{ \pm}=1$. Thus,

$$
k_{c}^{ \pm}=\frac{k_{\perp}^{4} A^{ \pm}\left(k_{\perp}, 0\right)}{v_{A}^{2}} .
$$




\subsection{The energy cascade time}

When $\bar{k}_{\|}^{-} \gg k_{c}^{-}$, the value of $\chi_{k_{\perp}}^{-}$is $\ll 1$ and a $w^{+}$is only weakly affected by a single collision with a $w^{-}$wave packet. Each such collision requires a time $\left(\bar{k}_{\|}^{-} v_{A}\right)^{-1}$. The effects of successive collisions add incoherently, and thus $\left(\chi_{k_{\perp}}^{-}\right)^{-2}$ collisions are required for the $w^{+}$wave packet to be strongly distorted, and for its energy to pass to smaller scales. The cascade time $\tau_{k_{\perp}}^{+}$for a $w^{+}$wave packet at perpendicular scale $k_{\perp}^{-1}$ is thus roughly

$$
\tau_{k_{\perp}}^{+} \sim\left(\bar{k}_{\|}^{-} v_{A}\right)^{-1}\left(\chi_{k_{\perp}}^{-}\right)^{-2} \sim \frac{1}{k_{c}^{-} v_{A}} \quad \text { (weak turbulence) }
$$

Similarly, if $\chi_{k_{\perp}}^{+} \ll 1$, then $\tau_{k_{\perp}}^{-} \sim\left(k_{c}^{+} v_{A}\right)^{-1}$.

When $\bar{k}_{\|}^{-} \sim k_{c}^{-}$, the value of $\chi_{k_{\perp}}^{-}$is $\sim 1$, a $w^{+}$is strongly distorted during a single wave packet collision, and the turbulence is strong. Each such collision takes a time $\left(\bar{k}_{\|}^{-} v_{A}\right)^{-1}$. Since $\bar{k}_{\|}^{-} \sim k_{c}^{-}$,

$$
\tau_{k_{\perp}}^{+} \sim\left(\bar{k}_{\|}^{-} v_{A}\right)^{-1} \sim \frac{1}{k_{c}^{-} v_{A}} \quad \text { (strong turbulence) }
$$

Similarly, if $\chi_{k_{\perp}}^{+} \sim 1$, then $\tau_{k_{\perp}}^{-} \sim\left(k_{c}^{+} v_{A}\right)^{-1}$.

The case $\bar{k}_{\|}^{ \pm} \ll k_{c}^{ \pm}$(i.e., $\chi_{k_{\perp}}^{ \pm} \gg 1$ ) is explicitly excluded from the discussion. Initial conditions could in principle be set up in which $\bar{k}_{\|}^{ \pm} \ll k_{c}^{ \pm}$. However, the cascade mechanisms described in section 3.2 will not produce the condition $\bar{k}_{\|}^{ \pm} \ll k_{c}^{ \pm}$if it is not initially present. It should be emphasized that in both weak turbulence and strong turbulence, the cascade time is given by the same formula, $\tau_{k_{\perp}}^{ \pm} \sim\left(k_{c}^{\mp} v_{A}\right)^{-1}$, which involves the $A^{\mp}$ spectrum evaluated only at $k_{\|}=0$.

\subsection{The Cascade of Energy to Larger $k_{\|}$}

The two basic mechanisms for transferring fluctuation energy to larger $k_{\|}$were identified by Lithwick, Goldreich, \& Sridhar (2007). The first of these can be called "propagation with distortion." Suppose a $w^{+}$wave packet of perpendicular scale $k_{\perp}^{-1}$ and arbitrarily large initial parallel correlation length begins colliding at $t=0$ with a stream of $w^{-}$wave packets of similar perpendicular scale. At time $t=\tau_{k_{\perp}}^{+}$, the leading edge of the $w^{+}$wave packet has been distorted substantially by the stream of $w^{-}$wave packets, but the trailing portion of the $w^{+}$wave packet at distances $\gtrsim 2 v_{A} \tau_{k_{\perp}}^{+}$behind the leading edge has not yet encountered the stream of $w^{-}$wave packets. If the parallel correlation length of the $w^{+}$wave packet is initially $>2 v_{A} \tau_{k_{\perp}}^{+}$, then during a time $\tau_{k_{\perp}}^{+}$the $w^{+}$wave packet acquires a spatial variation in the direction of the background magnetic field of length scale $\sim 2 v_{A} \tau_{k}^{+} \sim 2\left(k_{c}^{-}\right)^{-1}$. This process is modeled as diffusion of $w^{ \pm}$ 
fluctuation energy in the $k_{\|}$direction with diffusion coefficient $D_{\|}^{ \pm} \sim\left(\Delta k_{\|}\right)^{2} / \Delta t$, where $\Delta k_{\|}=k_{c}^{\mp}$ and $\Delta t=\tau_{k}^{ \pm}$. "Propagation with distortion" then leads to a value of $D_{\|}^{ \pm}$of $\sim\left(k_{c}^{\mp}\right)^{3} v_{A}$.

The second mechanism identified by Lithwick, Goldreich, \& Sridhar (2007) can be called "uncorrelated cascade." Consider a $w^{+}$wave packet of perpendicular scale $k_{\perp}^{-1}$ and arbitrarily large parallel correlation length, and consider two points within the wave packet, $P_{1}$ and $P_{2}$, that move with the wave packet at velocity $-v_{A} \hat{z}$ and are separated by a distance along $B_{0}$ of $2 v_{A} \tau_{k_{\perp}}^{-} \sim$ $2\left(k_{c}^{+}\right)^{-1}$. The $w^{-}$wave packets at perpendicular scale $k_{\perp}^{-1}$ encountered by the portions of the $w^{+}$wave packet at $P_{1}$ and $P_{2}$ are then uncorrelated, because $w^{-}$wave packets are substantially distorted while propagating between $P_{1}$ and $P_{2}$. Thus, the way in which the $w^{+}$wave packet cascades at location $P_{1}$ is not correlated with the way in which the $w^{+}$wave packet cascades at location $P_{2}$. If the parallel correlation length of the $w^{+}$wave packet is initially $>2 v_{A} \tau_{k_{\perp}}^{-}$, then wave-packet collisions introduce a spatial variation along $B_{0}$ into the $w^{+}$wave packet of length scale $\sim 2 v_{A} \tau_{k_{\perp}}^{-} \sim 2\left(k_{c}^{+}\right)^{-1}$ during a time $\tau_{k_{\perp}}^{+}$. Again, we model this as diffusion of $w^{ \pm}$fluctuation energy in the $k_{\|}$direction with $D_{\|}^{ \pm} \sim\left(\Delta k_{\|}\right)^{2} / \Delta t$ and $\Delta t=\tau_{k_{\perp}}^{ \pm}$, but now $\Delta k_{\|}=k_{c}^{ \pm}$. "Uncorrelated cascade" thus leads to a $k_{\|}$-diffusion coefficient of $\sim\left(k_{c}^{ \pm}\right)^{2} k_{c}^{\mp} v_{A}$.

Accounting for both mechanisms, one can write

$$
D_{\|}^{ \pm} \sim\left(k_{c, \max }\right)^{2} k_{c}^{\mp} v_{A},
$$

where $k_{c, \text { max }}\left(k_{\perp}\right)$ is the larger of $k_{c}^{+}\left(k_{\perp}\right)$ and $k_{c}^{-}\left(k_{\perp}\right)$. If $k_{c}^{+}>k_{c}^{-}$, then $w^{+}$energy diffuses in $k_{\|}$ primarily through the "uncorrelated cascade" mechanism, while $w^{-}$energy diffuses in $k_{\|}$primarily through the "propagation with distortion cascade" mechanism.

\subsection{Advection-Diffusion Model for the Power Spectra}

The phenomenology described in the preceding sections is encapsulated by the following nonlinear advection-diffusion equation,

$$
\frac{\partial A_{k}^{ \pm}}{\partial t}=-\frac{1}{k_{\perp}} \frac{\partial}{\partial k_{\perp}}\left(\frac{c_{1} k_{\perp}^{2} A_{k}^{ \pm} h_{k}^{ \pm}}{\tau_{\mathrm{eff}, k_{\perp}}^{ \pm}}\right)+c_{2}\left(k_{c, \max }\right)^{2} k_{c}^{\mp} v_{A} \frac{\partial^{2} A_{k}^{ \pm}}{\partial k_{\|}^{2}}+S_{k}^{ \pm}-\gamma_{k}^{ \pm} A_{k}^{ \pm},
$$

where $A_{k}^{ \pm}$is shorthand for $A^{ \pm}\left(k_{\perp}, k_{\|}\right), c_{1}$ and $c_{2}$ are dimensionless constants of order unity, and $S_{k}^{ \pm}$and $-\gamma_{k}^{ \pm} A_{k}^{ \pm}$are forcing and damping terms, respectively. The first term on the right-hand side of equation (15) represents advection of fluctuation energy to larger $k_{\perp}$, while the second term represents diffusion of fluctuation energy to larger $\left|k_{\|}\right|$. The quantity $\tau_{\mathrm{eff}, k_{\perp}}^{ \pm}$is an effective cascade time at perpendicular scale $k_{\perp}^{-1}$. Usually, the transfer of energy to small scales is dominated by

local interactions in $k$-space, and the cascade time for a $w^{+}$wave packet is $\sim\left(k_{c}^{\mp} v_{A}\right)^{-1}$. In some 
cases, however, the shearing of small-scale wave packets by much larger-scale wave packets can become important. To account for such cases, the effective cascade time is taken to be

$$
\left(\tau_{\mathrm{eff}, k_{\perp}}^{ \pm}\right)^{-1}=\max \left[\frac{q_{\perp}^{4} A^{\mp}\left(q_{\perp}, 0\right)}{v_{A}}\right] \quad \text { for } 0<q_{\perp}<k_{\perp},
$$

i.e., $\left(\tau_{\mathrm{eff}, k_{\perp}}^{ \pm}\right)^{-1}$ is the maximum value of $k_{c}^{\mp} v_{A}$ for all perpendicular wave numbers between zero and $k_{\perp}$. The flux of $w^{ \pm}$energy to larger $k_{\perp}$ is

$$
\varepsilon^{ \pm}\left(k_{\perp}\right)=2 \pi \int_{-\infty}^{\infty} d k_{\|} \frac{c_{1} k_{\perp}^{2} A_{k}^{ \pm} h_{k}^{ \pm}}{\tau_{\mathrm{eff}, k_{\perp}}^{ \pm}}
$$

The term $h_{k}^{ \pm}$is given by

$$
h_{k}^{ \pm}=-\frac{1}{A^{ \pm}\left(k_{\perp}, 0\right)} \frac{\partial}{\partial k_{\perp}}\left[k_{\perp} A^{ \pm}\left(k_{\perp}, 0\right)\right]
$$

and is included so that $\varepsilon^{ \pm}$increases as the $A^{ \pm}$spectrum becomes a more steeply declining function of $k_{\perp}$, in accordance with weak turbulence theory (Galtier et al 2000, Lithwick \& Goldreich 2003). To match the energy flux in weak turbulence theory in the limit of zero cross helicity, one must set 1

$$
c_{1}=-\frac{\pi J}{2}
$$

where

$$
J=\int_{1}^{\infty} d x \int_{-1}^{1} d y \frac{2\left[\left(x^{2}-1\right)\left(1-y^{2}\right)\right]^{1 / 2}(1+x y)^{2}\left[8-(x+y)^{3}\right] \ln [(x+y) / 2]}{\left(x^{2}-y^{2}\right)^{4}} \simeq-1.87 .
$$

For simplicity,

$$
c_{2}=1
$$

\section{Steady-State Weak Turbulence}

This section addresses weak turbulence in which $\bar{k}_{\|}^{+} \sim \bar{k}_{\|}^{-}$at the outer scale. The weakturbulence condition, $\chi_{k_{\perp}}^{ \pm} \ll 1$, is equivalent to the condition $k_{c}^{ \pm} \ll \bar{k}_{\|}^{ \pm}$. Because $k_{\|}$-diffusion

\footnotetext{
${ }^{1}$ The value of $c_{1}$ in equation (19) is a factor of 2 larger than the value that follows from the results of Galtier et al (2000). It appears that this discrepancy results from the omission of a factor of 2 in equation (54) of Galtier et al (2000). This can be seen by starting from equation (46) of Galtier et al (2000) and using the expression on page 1045 of Leith \& Kraichnan (1972) to simplify polar integrals of the form $\int d^{2} p d^{2} q \delta(k-p-q) F(k, p, q)$ for two-dimensional wave vectors $k, p$, and $q$, where $F$ is a function only of the wave-vector magnitudes and the integral is over all values of $p$ and $q$.
} 
involves a $\Delta k_{\|} \sim k_{c}^{ \pm}$during a time $\tau_{k_{\perp}}^{ \pm}$, the $k_{\|}$-increment over which energy diffuses while cascading to larger $k_{\perp}$ is much less than the breadth of the spectrum in the $k_{\|} \operatorname{direction}\left(\sim \bar{k}_{\|}^{ \pm}\right)$, so the $k_{\|}$diffusion terms can be ignored to a good approximation. In this case, equation (15) possesses a steady-state solution in which $\varepsilon^{+}$and $\varepsilon^{-}$are constant, and in which

$$
A_{k}^{ \pm}=g^{ \pm}\left(k_{\|}\right) k_{\perp}^{-n^{ \pm}}
$$

where $g^{+}$and $g^{-}$are arbitrary functions of $k_{\|}$, and where

$$
n^{+}+n^{-}=6
$$

with $2<n^{ \pm}<4$. Equations (22) and (23) match the results of weak turbulence theory for incompressible MHD turbulence if one allows only for three-wave interactions among shear Alfvén waves (Galtier \& Chandran 2006), or if one considers only the limit that $k_{\perp} \gg k_{\|}$(Galtier et al 2002). If one writes $n^{ \pm}=3 \pm \alpha$ with $|\alpha|<1$ and sets $g^{+}\left(k_{\|}\right)=g^{-}\left(k_{\|}\right)$, then equation (17) gives

$$
\frac{\varepsilon^{+}}{\varepsilon^{-}}=\frac{2+\alpha}{2-\alpha}
$$

In the limit $\alpha \ll 1, \varepsilon^{+} / \varepsilon^{-}=1+\alpha$, in agreement with the weak-turbulence-theory result for $k_{\perp} \gg\left|k_{\|}\right|$(Lithwick \& Goldreich 2003), as in the weak-turbulence advection-diffusion model of Lithwick \& Goldreich (2003).

In steady state, $A^{+}\left(k_{\perp}, 0\right)$ and $A^{-}\left(k_{\perp}, 0\right)$ are forced to be equal at the dissipation scale so that $\tau_{k}^{+}=\tau_{k}^{-}$. This phenomenon of "pinning" was discovered by Grappin et al (1983) for strong MHD turbulence, and further elaborated upon by Lithwick \& Goldreich (2003) for the case of weak turbulence. The dominant fluctuation type then has the steeper spectrum. If $\varepsilon^{+} / \varepsilon^{-}$is fixed, then the ratio $w_{k_{f}}^{+} / w_{k_{f}}^{-}$of the rms amplitudes of the two fluctuation types at the outer scale $k_{f}^{-1}$ increases as $k_{d} / k_{f}$ increases, where $k_{d}$ is the dissipation wave number. Alternatively, if $w_{k_{f}}^{+} / w_{k_{f}}^{-}$is fixed, then $\varepsilon^{+} / \varepsilon^{-}$approaches unity as $k_{d} / k_{f} \rightarrow \infty$.

Several of these results are illustrated by the numerical solution to equation (15) shown in Figure 2. This solution is obtained using a logarithmic grid for $k_{\perp}$, with $k_{\perp, i}=k_{0} 2^{i / n}$ for $0<i<N$. Similarly, $k_{\|, j}=k_{0} 2^{j / n}$ for $1<j<M$, but $k_{\|, j}=0$ for $j=0$. $A_{k}^{ \pm}$is advanced forward in time using a semi-implicit algorithm, in which the terms $h_{k}^{ \pm}, \tau_{\mathrm{eff}, k_{\perp}}^{ \pm}$, and $k_{c}^{ \pm}$on the right-hand side of equation (15) are evaluated at the beginning of the time step, and the $A^{ \pm}$terms on the right-hand side of equation (15) are evaluated at the end of the time step. The algorithm employs operator splitting, treating the $k_{\perp}$-advection, forcing, and damping in one stage, and the $k_{\|}$-diffusion is a second stage. In this approach, the matrix that has to be inverted to execute each semi-implicit time step is tri-diagonal. An advantage of this procedure over a fully explicit method is that the time step is not limited by the $k_{\|}$-diffusion time at large $k_{\perp}$ and small $k_{\|}$. The discretized equations 
are written in terms of the energy fluxes between neighboring cells, so that in the absence of forcing and dissipation the algorithm conserves fluctuation energy to machine accuracy. For the numerical solution plotted in Figure 2, $N=80, M=16, n=4, S^{ \pm}=S_{0}^{ \pm} k^{2} \exp \left(-k^{2} / k_{f}^{2}\right), S_{0}^{+}=1.2 S_{0}^{-}, k_{f}=$ $5 k_{0}$, and $\gamma_{k}^{ \pm}=2 k^{2} v$, where $v$ is an effective viscosity. The initial spectra are set equal to zero, and the equations are integrated forward in time until a steady state is reached. In steady state, $\delta v^{+}=2.5 \times 10^{-3} v_{A}$ and $\delta v^{-}=6.4 \times 10^{-4} v_{A}$.
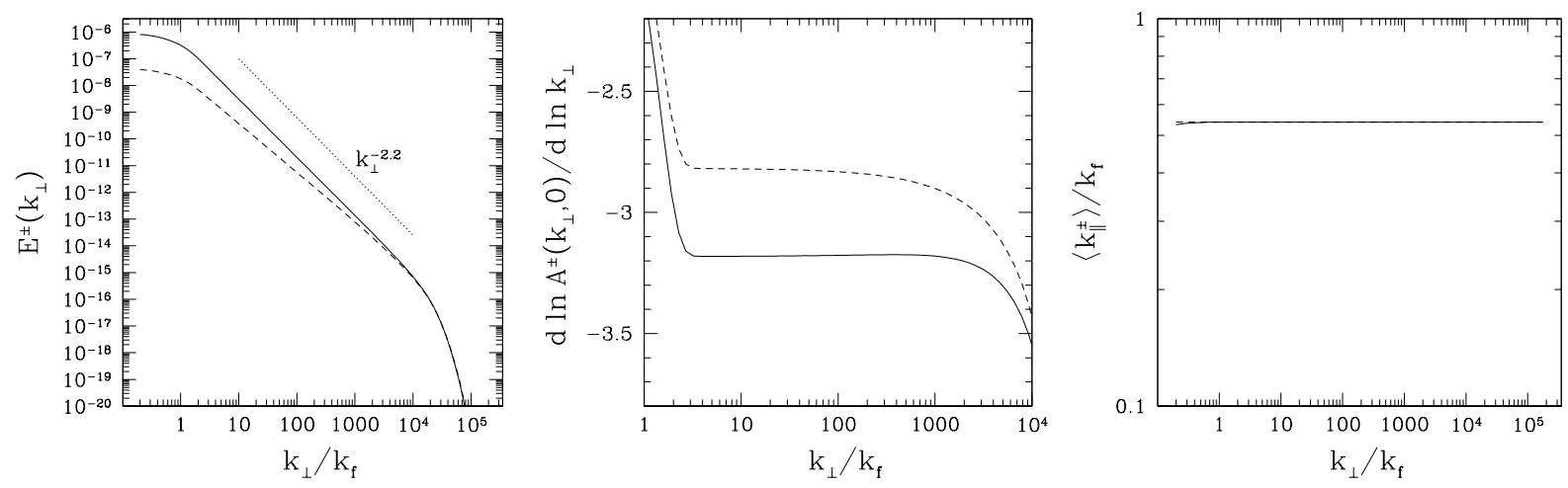

Fig. 2.- Numerical solution of equation (15) in the weak-turbulence limit. Left panel: The dimensionless 1D power spectrum defined in equation (25). Middle panel: The spectral slopes at $k_{\|}=0$. Right panel: The weighted value of $k_{\|}$ defined in equation (26). In all panels, the solid lines refer to $w^{+}$and the dashed lines refer to $w^{-}$. In the right-hand panel, the two lines are almost on top of each other.

The left-hand panel of Figure 2 is a plot of the dimensionless one-dimensional power spectrum,

$$
E^{ \pm}\left(k_{\perp}\right)=\frac{k_{0} k_{\perp}}{v_{A}^{2}} \int_{-\infty}^{\infty} d k_{\|} A^{ \pm}\left(k_{\perp}, k_{\|}\right),
$$

which is proportional to the energy per unit $k_{\perp}$ in $w^{ \pm}$fluctuations. The middle panel of Figure 2 shows that in the inertial range, $d \ln A^{+}\left(k_{\perp}, 0\right) / d \ln k \simeq-3.2$ and $d \ln A^{-}\left(k_{\perp}, 0\right) / d \ln k \simeq-2.8$, as expected for $S^{+} / S^{-}=1.2$. The right-hand panel shows that the weighted value of $k_{\|}$,

$$
\left\langle k_{\|}^{ \pm}\right\rangle=\frac{\int_{-\infty}^{\infty} d k_{\|}\left|k_{\|}\right| A^{ \pm}\left(k_{\perp}, k_{\|}\right)}{\int_{-\infty}^{\infty} d k_{\|} A^{ \pm}\left(k_{\perp}, k_{\|}\right)},
$$

is roughly constant in the inertial range. 


\section{Steady-State Strong Turbulence}

This section addresses strong turbulence with $\chi_{k_{f}}^{+} \sim 1, \chi_{k_{f}}^{-} \lesssim 1, w_{k_{f}}^{+} \geq w_{k_{f}}^{-}$, and $\bar{k}_{\|}^{+} \sim \bar{k}_{\|}^{-}$at the outer scale $k_{f}^{-1}$. The discussion allows for the possibility that $\chi_{k_{\perp}}^{-} \ll 1$. As fluctuation energy cascades to larger $k_{\perp}$, it diffuses to larger $\left|k_{\|}\right|$, so that $\bar{k}_{\|}^{+}$and $\bar{k}_{\|}^{-}$increase with increasing $k_{\perp}$. Moreover, for both $w^{+}$and $w^{-}$, the fluctuation energy diffuses over a $k_{\|}$-increment of $\sim k_{c}^{+}$during one cascade time. For the steady-state solutions of interest, $k_{c}^{+}$is an increasing function of $k_{\perp}$, and thus at each $k_{\perp}$ we will have that $\bar{k}_{\|}^{+} \sim \bar{k}_{\|}^{-} \sim k_{c}^{+}$. One can thus define a single parallel-wavenumber, $\overline{k_{\|}}\left(k_{\perp}\right)$, to describe the spectra, with

$$
\overline{k_{\|}} \sim \bar{k}_{\|}^{+} \sim \bar{k}_{\|}^{-} \sim k_{c}^{+}
$$

at each $k_{\perp}$. Since $\bar{k}_{\|}^{+} \sim k_{c}^{+}$at each scale,

$$
\chi_{k_{\perp}}^{+} \sim 1
$$

throughout the inertial range. On the other hand, since $w_{k_{\perp}}^{-}$can be much less than $w_{k_{\perp}}^{+}, \chi_{k_{\perp}}^{-}$can be $\ll 1$.

The cascade time for the $w^{-}$fluctuations is given by the strong-turbulence phenomenology of equation (13), so that the energy flux in $w^{-}$fluctuations is

$$
\varepsilon^{-} \sim \frac{\left(w_{k_{\perp}}^{-}\right)^{2}}{\tau_{k_{\perp}}^{-}} \sim k_{\perp} w_{k_{\perp}}^{+}\left(w_{k_{\perp}}^{-}\right)^{2} .
$$

Allowing for the possibility that $\chi_{k_{\perp}}^{-} \ll 1$, the cascade time of the $w^{+}$fluctuations follows the weak-turbulence phenomenology of equation (12). This formula is also accurate for $\chi_{k_{\perp}}^{-}$as large as 1 (in which case $w_{k_{\perp}}^{-} \sim w_{k_{\perp}}^{+}$). The energy flux in $w^{+}$fluctuations is then

$$
\varepsilon^{+} \sim \frac{\left(w_{k_{\perp}}^{+}\right)^{2}}{\tau_{k_{\perp}}^{+}} \sim \frac{k_{\perp}^{2}\left(w_{k_{\perp}}^{-}\right)^{2}\left(w_{k_{\perp}}^{+}\right)^{2}}{\overline{k_{\|}} v_{A}} \sim k_{\perp} w_{k_{\perp}}^{+}\left(w_{k_{\perp}}^{-}\right)^{2}
$$

which is roughly the same as $\varepsilon^{-}$. It is assumed that the energy flux depends on the spectral slope as in weak turbulence, so that the fluctuation type with the steeper spectrum has the larger energy flux. If

$$
w^{ \pm} \propto k_{\perp}^{-a^{ \pm}}
$$

then equations (29) and (30) imply that when $\varepsilon^{+}$and $\varepsilon^{-}$are independent of $k_{\perp}$,

$$
a^{+}+2 a^{-}=1
$$


The condition that $\chi_{k_{\perp}}^{+} \sim 1$ throughout the inertial range then implies that

$$
\overline{k_{\|}} \propto k_{\perp}^{1-a^{+}} .
$$

As discussed in earlier studies (Grappin et al 1983, Lithwick \& Goldreich 2003), the spectra are pinned at the dissipation scale, so that the dominant fluctuation type will have the steeper spectrum and a somewhat larger energy flux. For the zero-cross-helicity case, equations (32) and (33) give $w_{k_{\perp}}^{+}=w_{k_{\perp}}^{-} \propto k_{\perp}^{-1 / 3}$ and $\bar{k}_{\|} \propto k_{\perp}^{2 / 3}$, as in the work of Goldreich \& Sridhar (1995) [see also Higdon (1984)].

When $S_{k}^{ \pm}=\gamma_{k}^{ \pm}=0$, equation (15) possesses an analytical solution that reproduces the above scalings. This solution can be obtained by starting with the assumptions that

$$
A^{ \pm}\left(k_{\perp}, 0\right)=c_{3}^{ \pm} k_{\perp}^{-b^{ \pm}},
$$

that the energy cascade is dominated by local interactions, and that $A^{+}\left(k_{\perp}, 0\right)>A^{-}\left(k_{\perp}, 0\right)$ for all $k_{\perp}$. Equation (16) then becomes $\left(\tau_{\text {eff, } k_{\perp}}^{ \pm}\right)^{-1}=k_{\perp}^{4} A^{\mp}\left(k_{\perp}, 0\right) / v_{A}$, and $k_{c, \max }=k_{c}^{+}$. Upon defining

$$
f_{k}^{ \pm}=k_{\perp}^{6-b^{\mp}} A_{k}^{ \pm}
$$

and

$$
s=k_{\perp}^{8-2 b^{+}},
$$

one can rewrite equation (15) as

$$
\frac{\partial f_{k}^{ \pm}}{\partial s}=D^{ \pm} \frac{\partial^{2} f_{k}^{ \pm}}{\partial k_{\|}^{2}}
$$

with

$$
D^{ \pm}=\frac{c_{2}\left(c_{3}^{+}\right)^{2}}{c_{1}\left(8-2 b^{+}\right)\left(b^{ \pm}-1\right) v_{A}^{4}} .
$$

Equation (37) is solved by taking

$$
f_{k}^{ \pm}=\frac{c_{4}^{ \pm}}{\sqrt{s}} \exp \left(-\frac{k_{\|}^{2}}{4 D^{ \pm} s}\right) .
$$

Requiring that equation (34) be satisfied, one finds that $c_{4}^{ \pm}=c_{3}^{ \pm}$and

$$
2 b^{+}+b^{-}=10 .
$$

The dominance of local interactions requires that $b^{+}<4$, and thus $b^{-}>2$. When forcing and dissipation are taken into account, the exact solution becomes an approximate solution that is valid only within the inertial range. In this case, $b^{+}>b^{-}$because the spectra are pinned at the dissipation 
scale whereas $A^{+}\left(k_{\perp}, 0\right)$ is larger than $A^{-}\left(k_{\perp}, 0\right)$ within the inertial range. Equation (39) implies that

$$
\bar{k}_{\|}^{+} \simeq \bar{k}_{\|}^{-} \sim \sqrt{D^{ \pm} s} \sim \frac{c_{3}^{+} k_{\perp}^{4-b^{+}}}{v_{A}^{2}},
$$

where the dimensionless constants in the expression for $D^{ \pm}$have been dropped, but $c_{3}^{+}$, which has dimensions, has been kept. Equations (11), (34), and (41) show that $k_{c}^{+} \sim \bar{k}_{\|}^{+}$for all $k_{\perp}$, so that $\chi_{k_{\perp}}^{+} \sim 1$ for all $k_{\perp}$. Equation (25) gives

$$
E^{ \pm}\left(k_{\perp}\right) \propto A^{ \pm}\left(k_{\perp}, 0\right) k_{\perp} \bar{k}_{\|}^{+} \propto k_{\perp}^{5-b^{+}-b^{ \pm}},
$$

from which it follows that

$$
w_{k_{\perp}}^{+} \propto k_{\perp}^{3-b^{+}}
$$

and

$$
w_{k_{\perp}}^{-} \propto k_{\perp}^{\left(6-b^{+}-b^{-}\right) / 2} .
$$

This solution reduces to the critical-balance solution of Goldreich \& Sridhar (1995) when $b^{+}=$ $b^{-}=10 / 3$, in which case $\bar{k}_{\|}^{ \pm} \propto k_{\perp}^{2 / 3}$, and $w_{k_{\perp}}^{ \pm} \propto k_{\perp}^{-1 / 3}$. Comparing equations (43) and (44) with equation (31), it can be seen that $b^{+}$corresponds to $3+a^{+}$and $b^{-}$corresponds to $3+2 a^{-}-a^{+}$. Equation (41) is thus equivalent to equation (33), and equation (40) is equivalent to equation (32).

Figure 3 shows the results from a numerical solution of equation (15), obtained by integrating forward in time as described in section 4 with the spectra initially equal to zero. The numerical solution was obtained by setting $S^{ \pm}=S_{0}^{ \pm} k^{2} \exp \left(-k^{2} / k_{f}^{2}\right)$ with $S_{0}^{+}=1.2 S_{0}^{-}$and using the parameters (defined in section 4) $N=80, M=60, n=4, k_{f}=5 k_{0}$, and $\gamma_{k}^{ \pm}=2 k^{2} v$, where the constant $v$ is an effective viscosity. The rms velocities at steady state are $\delta v^{+}=1.5 v_{A}$ and $\delta v^{-}=0.10 v_{A}$. The lefthand panel shows that the one-dimensional energy spectrum $E_{k_{\perp}}^{+}$is $\propto k_{\perp}^{-2.14}$ in the inertial range, which corresponds to $b^{+}=3.57$ in equation (42). Equation (40) then gives $b^{-}=2.86$. The dotted lines in the middle panel of Figure 3 correspond to the values of $b^{+}=3.57$ and $b^{-}=2.86$, which are reasonably close to the values of $-d \ln A^{ \pm}\left(k_{\perp}, 0\right) / d \ln k_{\perp}$ in the numerical solution, although these latter values vary throughout the inertial range in the numerical solution. For $b^{+}=3.57$, equation (41) gives $\bar{k}_{\|}^{+} \propto k_{\perp}^{0.43}$, which is a close match to the numerical solution, as shown in the right-hand panel of Figure 3. The left-hand panel of Figure 3 shows that the steady-state solutions for $A^{+}$and $A^{-}$are "pinned" at the dissipation scale, as expected.

It should be noted that when $\chi_{k_{\perp}}^{+} \sim 1$ and $\chi_{k_{\perp}}^{-} \ll 1$, the dominant $w^{+}$fluctuations are only weakly damped by nonlinear interactions with $w^{-}$waves, in the sense that $\tau_{k_{\perp}}^{+}$is much larger than the linear wave period. On the other hand, for the smaller-amplitude $w^{-}$fluctuations, the linear wave period and cascade time are comparable. Thus, paradoxically, the larger-amplitude $w^{+}$ fluctuations can be described as waves, or, more precisely, a non-sinusoidal wave train, whereas the smaller-amplitude $w^{-}$fluctuations can not be accurately described as waves. 

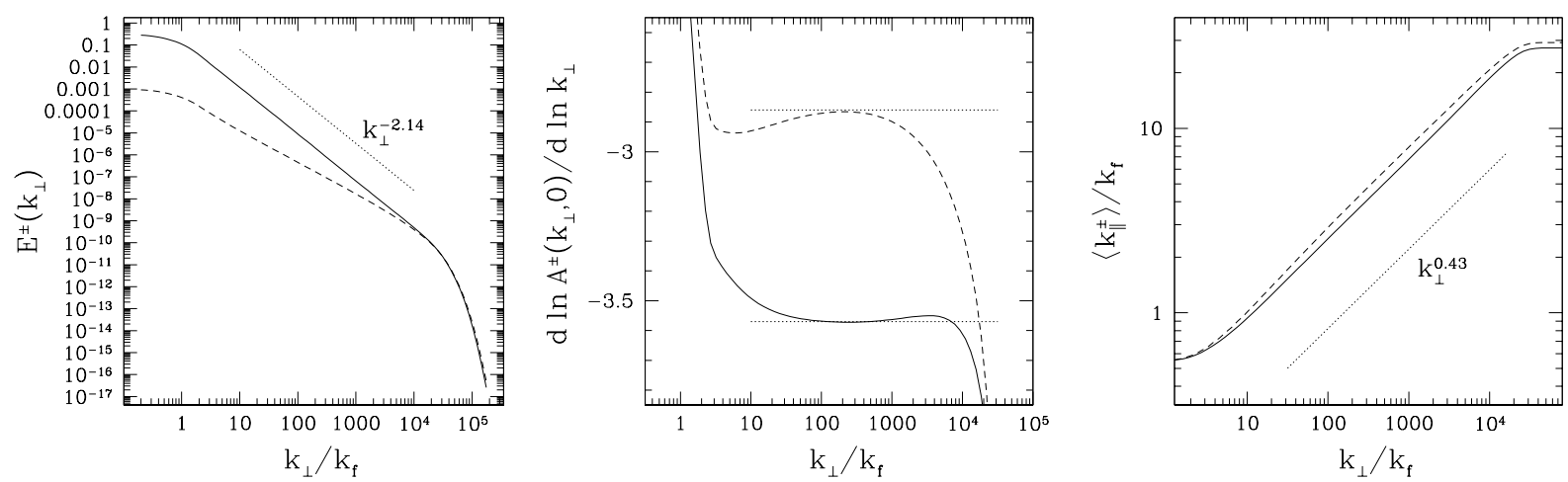

Fig. 3.- Numerical solution of equation (15) for strong turbulence with $\chi_{k_{\perp}}^{+} \sim 1$. Left panel: The dimensionless 1D power spectrum defined in equation (25). Middle panel: The spectral slopes at $k_{\|}=0$. Right panel: The weighted value of $k_{\|}$defined in equation (26). In all panels, the solid lines refer to $w^{+}$and the dashed lines refer to $w^{-}$.

\section{Transition Between Weak Turbulence and Strong Turbulence}

This section again addresses turbulence in which $\bar{k}_{\|}^{+} \sim \bar{k}_{\|}^{-}$at the outer-scale wavenumber, $k_{f}$. In the weak-turbulence limit, $\chi_{k_{\perp}}^{+}$and $\chi_{k_{\perp}}^{-}$increase with increasing $k_{\perp}$. If the dissipation wavenumber $k_{d}$ is sufficiently large, then $\chi_{k_{\perp}}^{+}$and/or $\chi_{k_{\perp}}^{-}$will increase to a value of order unity at some $k_{\perp}$ within the inertial range. This perpendicular wavenumber is denoted $k_{\text {trans. }}$. The turbulence will then be described by the weak-turbulence scalings of section 4 for $k_{f} \ll k_{\perp} \ll k_{\text {trans }}$, and by the strong-turbulence scalings of section 5 for $k_{\text {trans }} \ll k_{\perp} \ll k_{d}$. Figure 4 shows a numerical solution of equation (15) that illustrates how the turbulence makes this transition in a smooth manner. At small wavenumbers, this solution is similar to the weak-turbulence solution plotted in Figure 2, and at large wavenumbers it is similar to the strong-turbulence solution plotted in Figure 3, The solution shown in Figure 4 was obtained by integrating equation (15) forward in time to steady state using the numerical method described in section 4. The spectra were initially set equal to zero. The numerical solution was obtained by setting $S^{ \pm}=S_{0}^{ \pm} k^{2} \exp \left(-k^{2} / k_{f}^{2}\right)$ with $S_{0}^{+}=1.2 S_{0}^{-}$ and using the parameters $N=100, M=56, n=4, k_{f}=5 k_{0}$, and $\gamma_{k}^{ \pm}=2 k^{2} v$, where the constant $v$ is an effective viscosity. The rms velocities at steady state are $\delta v^{+}=0.32 v_{A}$ and $\delta v^{-}=0.012 v_{A}$.

\section{Unequal Parallel Correlation Lengths at the Outer Scale}

In sections 4 through 6, it was assumed that $\bar{k}_{\|}^{+} \sim \bar{k}_{\|}^{-}$at the outer scale. This assumption is applicable to many settings. For example, in a plasma of dimension $L$ that is stirred by a force that has a correlation length $l \ll L$, the velocity fluctuations that are excited have a correlation length $l$, and this correlation length is imprinted on both the $w^{+}$and $w^{-}$fluctuations. On the other 

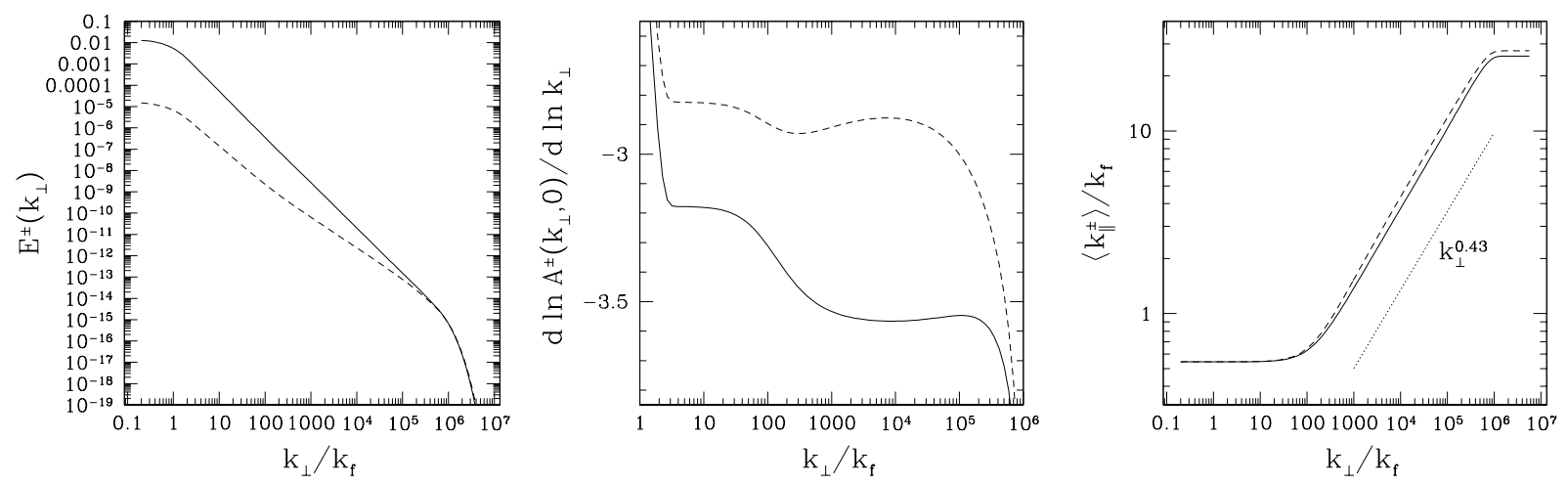

Fig. 4.- Numerical solution of equation (15) showing a smooth transition from the weak-turbulence limit at small $k_{\perp}$ to the strong-turbulence limit at large $k_{\perp}$. Left panel: The dimensionless 1D power spectrum defined in equation (25). Middle panel: The spectral slopes at $k_{\|}=0$. Right panel: The weighted value of $k_{\|}$defined in equation (26). In all panels, the solid lines refer to $w^{+}$and the dashed lines refer to $w^{-}$.

hand, if waves are launched along the magnetic field into a bounded plasma from opposite sides of the plasma, and the waves from one side have a much larger parallel correlation length than the waves from the other side, it is possible to set up turbulence in which the two wave types have very different parallel correlation lengths at the outer scale. This situation is discussed briefly in this section.

For strong turbulence, if both $\chi_{k_{\perp}}^{+}$and $\chi_{k_{\perp}}^{-}$are $\sim 1$ at some perpendicular scale $k_{\perp}^{-1}$, but one fluctuation type, say $w^{+}$, has a much smaller parallel correlation length than the other (and thus a much larger amplitude), then during a time $\tau_{k_{\perp}}^{-}$the "propagation with distortion" mechanism discussed in section 3.2 will increase $\bar{k}_{\|}^{-}$until it equals $\bar{k}_{\|}^{+}$, which will cause $\chi_{k_{\perp}}^{-}$to become $\ll 1$ at scale $k_{\perp}^{-1}$. At smaller scales, the solution can be described by the scalings presented in section 5 , in which $\bar{k}_{\|}^{+}\left(k_{\perp}\right) \sim \bar{k}_{\|}^{-}\left(k_{\perp}\right)$. Similarly, if $\chi_{k_{\perp}}^{+} \sim 1$ but $\chi_{k_{\perp}}^{-} \ll 1$ at some scale $k_{\perp}^{-1}$ and if $\bar{k}_{\|}^{+} \gg \bar{k}_{\|}^{-}$ at that scale, then during a time $\tau_{k_{\perp}}^{-}$the "propagation with distortion" mechanism discussed in section 3.2 will again increase $\bar{k}_{\|}^{-}$until it equals $\bar{k}_{\|}^{+}$, the parallel scales will remain comparable at smaller perpendicular scales, and the solution can be described by the scalings in section 5 , The case in which $\chi_{k_{\perp}}^{+} \sim 1, \chi_{k_{\perp}}^{-} \ll 1$, and $\bar{k}_{\|}^{+} \ll \bar{k}_{\|}^{-}$is not addressed in this paper.

\section{Implications for Turbulence in the Solar Corona and Solar Wind}

In this section, the preceding analysis of incompressible MHD turbulence is applied to the solar wind and solar corona. It should be noted at the outset, however, that the solar wind and solar corona (beyond roughly $r=1.5 R_{\odot}$, where $r$ is distance from the Sun's center) are in the 
collisionless regime, and the pressure tensor is not isotropic as assumed in ideal MHD. Moreover, the value of $\beta=8 \pi p / B^{2}$ is $\ll 1$ in the corona and typically $\sim 1$ in the solar wind at $1 \mathrm{AU}$, whereas incompressible MHD corresponds to the limit $\beta \rightarrow \infty$. A preliminary question that needs to be addressed is thus the extent to which incompressible MHD is an accurate model for these plasmas.

Schekochihin et al (2007) have recently carried out extensive calculations based on kinetic theory that provide a detailed answer to this question. These authors examined anisotropic turbulence in weakly collisional magnetized plasmas using gyrokinetics, a low-frequency expansion of the Vlasov equation that averages over the gyromotion of the particles. By applying the form of the gyrokinetic expansion derived by Howes et al (2006), Schekochihin et al (2007) showed analytically that non-compressive Alfvénic turbulence in the quasi-2D regime (i.e., $k_{\perp} \gg k_{\|}$) can be accurately described using reduced MHD in both the collisional and collisionless limits, regardless of $\beta$, provided that the length scales of the fluctuations are much larger than the proton gyroradius and the frequencies are much less than the proton cyclotron frequency. Since non-compressive quasi-2D fluctuations are thought to be the dominant component of the turbulence in the solar wind (see, e.g., Bieber et al 1994) and the solar corona (Dmitruk \& Matthaeus 2003, Cranmer \& van Ballegooijen 2005), incompressible MHD is a useful approximation for modeling turbulence in these settings.

\subsection{Cross helicity in the solar wind and solar corona}

Cross helicity in the solar wind has been measured in situ by several different spacecraft. In terms of the Elsasser variables $w^{ \pm}$, there is a substantial excess of outward propagating fluctuations (taken to be $w^{+}$throughout this section) over inward propagating fluctuations (taken to be $w^{-}$) in the inner heliosphere, although this imbalance decreases with increasing $r$, as seen, for example, in Voyager data for low heliographic latitude (Matthaeus \& Goldstein 1982, Roberts et al 1987) and Ulysses data at high latitude (Goldstein et al 1995). In a study of Ulysses and Helios data, Bavassano et al (2000) found that $e^{+} / e^{-} \propto r^{-1.02}$ for $r<2.6 \mathrm{AU}$ and $e^{+} / e^{-} \sim 2$ for $3 \mathrm{AU} \lesssim r \lesssim$ $5 \mathrm{AU}$, where $e^{ \pm}$is the energy per unit mass associated with $w^{ \pm}$fluctuations. These numbers are intended as illustrative average values, as individual measurements of $e^{+} / e^{-}$in the solar wind vary significantly.

Although it has not been directly measured, the ratio $e^{+} / e^{-}$is likely very large in open-fieldline regions of the solar corona. This can be seen from the work of Cranmer \& van Ballegooijen (2005), who modeled the generation of Alfvén waves by the observed motions of field-line foot-

points in the photosphere, and the propagation and reflection of these waves as they travel along open field lines from the photosphere out into the interplanetary medium. They found that the ratio of the frequency-integrated rms Elsasser variables $\left(w^{+}\right.$and $\left.w^{-}\right)$is $\sim 30$ at $r=2 R_{\odot}$ (i.e., 
$\left.e^{+} / e^{-} \sim 900\right)$. Verdini \& Velli (2007) developed a different model for the propagation, reflection, and turbulent dissipation of Alfvén waves in the solar atmosphere and solar wind and found that $e^{+} / e^{-} \simeq 80$ at $r=2 R_{\odot}$. Based on these results, one can make the rough estimate that

$$
\frac{w_{k_{f}}^{+}}{w_{k_{f}}^{-}} \sim 10 \quad\left(\text { at } r=2 R_{\odot}\right)
$$

where $k_{f}$ is the perpendicular wavenumber at the outer scale.

\subsection{Is quasi-2D turbulence in the corona and solar wind weak or strong?}

In much of the solar wind, $\delta B$ is comparable to $B_{0}$, and the turbulence is in the strongturbulence regime with $\chi_{k_{f}}^{+} \sim 1$. For the corona, Cranmer \& van Ballegooijen (2005) found that the outer-scale fluctuations in open-field-line regions have periods of $T=1-5$ minutes, $\delta v \sim 100 \mathrm{~km} / \mathrm{s}$, and perpendicular correlation lengths of $L_{\perp} \sim k_{f}^{-1} \sim 10^{4} \mathrm{~km}$. The Alfvén speed in their model corona is between 2000 and $3000 \mathrm{~km} / \mathrm{s}$ at $r=2 R_{\odot}$, and thus $\delta v \ll v_{A}$. However, the parallel correlation length $L_{\|}$of the outer-scale fluctuations is $\sim v_{A} T=1.8-9 \times 10^{5} \mathrm{~km}$, which is $\gg L_{\perp}$. Because $L_{\|} / L_{\perp} \sim v_{A} / \delta v$

$$
\chi_{k_{f}}^{+} \sim 1
$$

and the low-frequency fluctuations launched into the corona by footpoint motions are in the strongturbulence regime. There may be an additional population of higher-frequency waves in the weakturbulence regime, but these are not discussed here.

\subsection{Parallel correlation lengths of inward and outward waves}

In open-field-line regions of the corona, when $w^{+}$waves are reflected, the resulting $w^{-}$waves have the same frequencies as the $w^{+}$waves. On the other hand, wave-reflection is more efficient at lower frequencies (Velli 1993), so if there is a range of wave frequencies at each $k_{\perp}$, the energyweighted average frequency of inward waves would tend to be somewhat lower than that of the outward waves. This suggests that at the outer scale the parallel correlation length $L_{\|}$of the $w^{-}$ fluctuations is somewhat larger than the value of $L_{\|}$for the $w^{+}$fluctuations. However, given equation (46), the $w^{+}$fluctuations imprint their parallel correlation length on the $w^{-}$fluctuations during a single turnover time $\tau_{k_{\perp}}^{-}$, as argued in section 7. The parallel correlation lengths of the $w^{+}$ and $w^{-}$fluctuations in the corona can thus be taken to be approximately equal at the outer scale, and hence also at smaller scales. The same approximation is reasonable for turbulence in the solar wind. 


\subsection{Energy Dissipation Rate}

If we take $k_{f}$ to be the perpendicular wave number at the outer scale, $w_{k_{f}}^{+}$to be the rms amplitude of the outward-propagating fluctuations at the outer scale, and $w_{k_{f}}^{-}$to be the rms amplitude of the Sunward-propagating fluctuations at the outer scale, then equations (29), (30), and (46) imply that

$$
\varepsilon^{+} \sim \varepsilon^{-} \sim k_{f} w_{k_{f}}^{+}\left(w_{k_{f}}^{-}\right)^{2}
$$

This estimate is a factor of $\sim w_{k_{f}}^{-} / w_{k_{f}}^{+}$smaller than the standard strong-turbulence estimate of $\varepsilon^{+} \sim k_{f}\left(w_{k_{f}}^{+}\right)^{2} w_{k_{f}}^{-}$that appears in many studies (e.g., Zhou \& Matthaeus 1990, Cranmer \& Van Ballegooijen 2005, Lithwick, Goldreich, \& Sridhar 2007, Verdini \& Velli 2007). This difference has important implications for turbulent heating of the solar corona and solar wind.

\subsection{Cascade Time}

For the energetically dominant $w^{+}$fluctuations, the cascade time $\tau_{k_{\perp}}^{+}$is much longer than the linear wave period, at least at scales much larger than the dissipation scale. This result is important for determining the conditions under which turbulence can be a viable mechanism to explain the heating of the solar corona. Observations taken with the Ultraviolet Coronagraph Spectrometer (UVCS) indicate that there is strong heating of coronal plasma at $r \lesssim 2 R_{\odot}$ (Kohl et al 1998, Antonucci et al 2000). An appealing model to explain this heating is that low-frequency Alfvén waves are launched by turbulent motions of field-line footpoints in the photosphere, that some of these waves are reflected, and that interactions between oppositely directed Alfvén wave packets in the corona causes the wave energy to cascade to small scales and dissipate (Matthaeus et al 1999, 2002; Dmitruk et al 2001, 2002; Cranmer \& van Ballegooijen 2005, 2007; Verdini $\&$ Velli 2007). In one version of this model, the waves that cross the transition region and enter the corona have not yet undergone a turbulent cascade, and their energy is concentrated at the fairly long periods ( $>1$ minute) characteristic of the observed footpoint motions that are believed to make the dominant contribution to the outward directed wave flux. In order for this scenario to explain the UVCS measurements, there needs to be time for the outer-scale waves to cascade within the corona before they travel beyond $r \simeq 2 R_{\odot}$. If, as above, we take $k_{f}$ to be the value of $k_{\perp}$ at the outer scale, $w_{k_{f}}^{+}$to be the rms amplitude of the outward waves at the outer scale, and $L_{\|}$to be the parallel correlation length of the fluctuations at the outer scale, then equation (12) can be used to express the cascade time for the outward waves at the outer scale as

$$
\tau_{k_{f}}^{+} \sim \frac{L_{\|}}{v_{A}}\left(\frac{w_{k_{f}}^{+}}{w_{k_{f}}^{-}}\right)^{2}\left(\chi_{k_{f}}^{+}\right)^{-2},
$$


where $\chi_{k_{f}}^{+} \sim k_{f} w_{k_{f}}^{+} L_{\|} / v_{A}$. Thus, for waves with a period $L_{\|} / v_{A} \sim 1$ minute, equations (45), (46), and (48) give $\tau_{k_{f}}^{+} \sim 100$ minutes. On the other hand, the Alfvén speed in a coronal hole at $r<2 R_{\odot}$ is $\sim 2000-3000 \mathrm{~km} / \mathrm{s}$ (Cranmer \& van Ballegooijen 2005), and the time for an Alfvén wave to travel from the coronal base out to $r=2 R_{\odot}$ is $4-6$ minutes. There is thus not enough time for the energy of waves with periods $>1$ minute to cascade and dissipate within a few solar radii of the Sun.

Dmitruk \& Matthaeus (2003) and Verdini \& Velli (2007) avoid this difficulty by postulating that a broad frequency spectrum of waves is launched upwards from the photosphere. Another possible way around this difficulty is the development of a broad frequency spectrum of fluctuations from wave-packet collisions in the chromosphere, in which the energies of inward and outward propagating waves are comparable due to strong wave reflection at the transition region.

\subsection{Spectral Index}

Much of the discussion of the inertial-range power spectrum of solar-wind turbulence has focused on the question of whether the spectral index is closer to the Kolmogorov (1941) value of $-5 / 3$ or the Iroshnikov-Kraichnan value of $-3 / 2$ (Iroshnikov 1963, Kraichnan 1965). A value of $-5 / 3$ is supported by a number of theoretical studies (e.g., Montgomery \& Turner 1981, Higdon 1984, Goldreich \& Sridhar 1995) and numerical simulations (Cho \& Vishniac 2000, Müller \& Biskamp 2000, Cho et al 2002, Haugen et al 2004). A value value of $-3 / 2$ is supported by a second group of theoretical studies (Boldyrev 2005, 2006; Mason et al 2006; see also Beresnyak \& Lazarian 2006) and numerical simulations (Maron \& Goldreich 2001, Müller et al 2003, Müller \& Grappin 2005, Mininni \& Pouquet 2007). It should be noted that all of the above-mentioned studies address MHD turbulence with negligible cross helicity.

Spacecraft measurements yield frequency spectra for the magnetic field and velocity fluctuations, where the frequency $f$ is approximately $k_{r} U / 2 \pi$, where $k_{r}$ is the radial component of the wave-vector and $U$ is the solar-wind speed [Taylor's (1938) "frozen-in flow hypothesis"]. Below a spectral-break frequency $f_{b}$, the spectra are typically fairly flat, being approximately proportional to $f^{-1}$ (Matthaeus \& Goldstein 1986). At $f>f_{b}$, the spectra steepen. The time scale corresponding to the spectral break, $f_{b}^{-1}$, increases with increasing $r$. For example, Bruno \& Carbone (2005) found that $f_{b}^{-1}$ was 0.06 hours at $0.3 \mathrm{AU}, 0.16$ hours at $0.7 \mathrm{AU}$, and 0.4 hours at $0.9 \mathrm{AU}$ in a sample of Helios 2 data. In two other studies based on data from several spacecraft, Matthaeus \& Goldstein (1986) found that $f_{b}^{-1} \sim 3.5$ hours at $1 \mathrm{AU}$, while Klein et al (1992) found $f_{b}^{-1} \sim 12$ hours at 4 AU. The inertial range roughly corresponds to frequencies in the interval $f_{b}<f<f_{d}$, where $f_{d}$ is the frequency corresponding to the dissipation scale. At $1 \mathrm{AU}, f_{d} \sim 0.3 \mathrm{~s}^{-1}$ (Smith et al 2006). A large number of inertial-range spectral indices have been reported in the literature. For example, 
Matthaeus \& Goldstein (1982) found a spectral index of $-1.73 \pm 0.08$ for the magnetic field in Voyager data at $r=1 \mathrm{AU}$, and a spectral index of $-1.69 \pm 0.08$ for the total energy. Goldstein et al (1995, Fig. 1) found that the spectral index for the $w^{+}$fluctuations was slightly steeper than $-5 / 3$ in Ulysses data at $2 \mathrm{AU}$ and $4 \mathrm{AU}$. Their results also suggest a shallower $w^{-}$spectrum, consistent with the idea that the spectra are pinned at the dissipation wavenumber $k_{d}$. In a study of Helios 2 magnetic-field data, Bruno \& Carbone (2005, Figure 23) found spectral indices of -1.72 at $0.3 \mathrm{AU},-1.67$ at $0.7 \mathrm{AU}$, and -1.70 at $0.9 \mathrm{AU}$. Using data from the WIND spacecraft at $1 \mathrm{AU}$, Podesta et al (2007) found a total-energy spectral index of -1.63 , with the velocity spectrum flatter than the magnetic spectrum. Marsch \& Tu (1996) found a spectral index of $-1.65 \pm 0.01$ for the magnetic field at $1 \mathrm{AU}$ in Helios 2 data. Horbury \& Balogh (1995) found a spectral index close to $-5 / 3$ for the magnetic field in Ulysses data at 2.5 AU. Using magnetic-field data from the ACE spacecraft at $1 \mathrm{AU}$, Smith et al (2006) found a spectral index of $-1.63 \pm 0.14$ in open-field-line regions and $-1.56 \pm 0.16$ in magnetic clouds. Smith (2003) found spectral indices between -1.7 and -1.8 in a study of Ulysses magnetic-field data covering a range of heliographic latitudes and radii.

Overall, the spectra are more consistent with a Kolmogorov scaling than an IroshnikovKraichnan scaling. It should be emphasized, however, that the observations in several cases are consistent with inertial range spectra that are steeper than a Kolmogorov spectrum. Spectral indices $>5 / 3$ have been found in previous theoretical studies of weak incompressible MHD turbulence (Bhattacharjee \& Ng 1997, Goldreich \& Sridhar 1997, Galtier et al 2000, Bhattacharjee \& Ng 2001, Perez \& Boldyrev 2008), as well as strong isotropic MHD turbulence with cross helicity (Grappin et al 1983). In this paper, it is argued that spectral indices $>5 / 3$ are a consequence of cross helicity in strong anisotropic MHD turbulence.

The simplest way to apply this paper to solar wind turbulence is to model the solar wind fluctuations at some location as steady-state, forced, homogeneous turbulence with the same average value of $w_{k_{f}}^{+} / w_{k_{f}}^{-}$, where $w_{k_{f}}^{+}$and $w_{k_{f}}^{-}$are the rms amplitudes of the $w^{ \pm}$fluctuations at the outer scale $k_{f}^{-1}$. Upon setting $w_{k_{\perp}}^{ \pm} \propto k_{\perp}^{-a^{ \pm}}$, one can write $\left(w_{k_{\perp}}^{+} / w_{k_{\perp}}^{-}\right)^{2} \propto\left(k_{\perp} / k_{d}\right)^{-2 a^{+}+2 a^{-}}$, where it is assumed that the spectra are equal at the dissipation wave number $k_{d}$. Equation (32) then gives $\left(w_{k_{\perp}}^{+} / w_{k_{\perp}}^{-}\right)^{2} \simeq\left(k_{\perp} / k_{d}\right)^{1-3 a^{+}}$, and the value of $a^{+}$can be obtained from the equation $\left(w_{k_{f}}^{+} / w_{k_{f}}^{-}\right)^{2} \simeq\left(k_{f} / k_{d}\right)^{1-3 a^{+}}$. The total-energy spectrum, $E\left(k_{\perp}\right)=E^{+}\left(k_{\perp}\right)+E^{-}\left(k_{\perp}\right)$, is approximately $k_{\perp}^{-1}\left(w_{k_{\perp}}^{+}\right)^{2}$, although it is flatter than $k_{\perp}^{-1}\left(w_{k_{\perp}}^{+}\right)^{2}$ near the dissipation scale where the flatter spectrum of the $w^{-}$fluctuations is important. Thus, at scales much larger than the dissipation scale,

$$
E\left(k_{\perp}\right) \propto k_{\perp}^{-q},
$$

where

$$
q=\frac{5}{3}+\frac{2 \log _{10}\left[\left(w_{k_{f}}^{+}\right)^{2} /\left(w_{k_{f}}^{-}\right)^{2}\right]}{3 \log _{10}\left(k_{d} / k_{f}\right)}
$$


Upon defining the outer-scale fractional cross helicity as

$$
\sigma_{c}=\frac{\left(w_{k_{f}}^{+}\right)^{2}-\left(w_{k_{f}}^{-}\right)^{2}}{\left(w_{k_{f}}^{+}\right)^{2}+\left(w_{k_{f}}^{-}\right)^{2}}
$$

one can rewrite equation (50) as

$$
q=\frac{5}{3}+\frac{2 \log _{10}\left[\left(1+\sigma_{c}\right) /\left(1-\sigma_{c}\right)\right]}{3 \log _{10}\left(k_{d} / k_{f}\right)} .
$$

The spectral index from equation (52) is plotted in Figure 5, assuming that $k_{d} / k_{f}=f_{d} / f_{b}=3780$, where $f_{b}=(3.5 \text { hours })^{-1}$ is the break frequency at $1 \mathrm{AU}$ discussed above (Matthaeus \& Goldstein 1986) and $f_{d}=0.3 \mathrm{~s}^{-1}$ is the frequency at the dissipation scale. (Smith et al 2006) With this choice, $q=1.78$ for $\left(w_{k_{f}}^{+}\right)^{2} /\left(w_{k_{f}}^{-}\right)^{2}=4$ and $q=1.74$ for $\left(w_{k_{f}}^{+}\right)^{2} /\left(w_{k_{f}}^{-}\right)^{2}=2$. When equation (52) is applied to the solar wind, $\sigma_{c}$ should be interpreted as the cross helicity at the outer scale $k_{f}^{-1}$ averaged over at least a few outer-scale fluctuations.

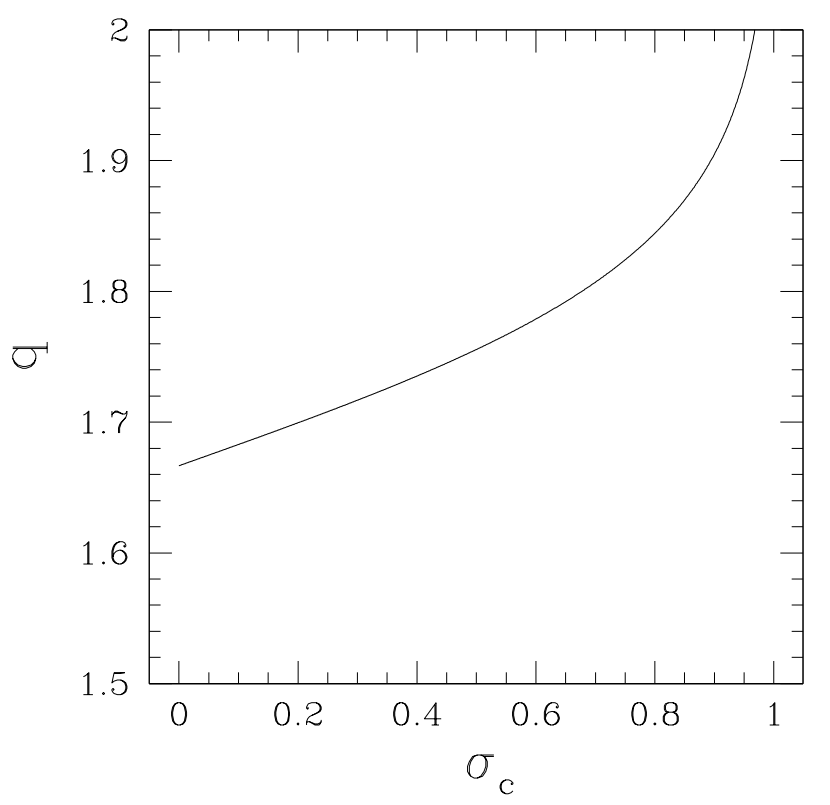

Fig. 5.- Dependence of spectral index of the total energy spectrum, $q$, on the outer-scale fractional cross helicity $\sigma_{c}$. The ratio of the dissipation wavenumber $k_{d}$ to the perpendicular wavenumber at the outer-scale $k_{f}$ in equation (52) is taken to be 3780 .

Some caution, however, is warranted when applying equation (52) to the solar wind because solar wind conditions vary with $r$, while equation (52) is based on results for homogeneous turbulence. The condition under which it is valid to treat the solar-wind fluctuations at a some $r$ with an 
observed $\sigma_{c}$ as homogeneous turbulence with the same $\sigma_{c}$ is that the cascade time at the outer scale $\tau_{k_{f}}^{ \pm}$be much smaller than the time $t_{\mathrm{adv}}=r / U$ for turbulent structures to be advected a distance $r$, where $U$ is the solar wind speed. However, this condition is often either violated or only marginally satisfied. This is illustrated by the results of Matthaeus \& Goldstein (1982) based on four days of Voyager data (in their Table 1). For the fluctuations at $1 \mathrm{AU}$, these authors found an rms velocity fluctuation of $\delta v=25.5 \mathrm{~km} / \mathrm{s}$ [which is comparable to $v_{A}$ at $1 \mathrm{AU}$ ], a velocity correlation length (outer scale) of $L_{\perp} \sim 2.83 \times 10^{11} \mathrm{~cm}$, and an average solar-wind speed of $352 \mathrm{~km} / \mathrm{s}$. In this particular data set, $\sigma_{c}=0.06$, so that $w_{k_{f}}^{+} \sim w_{k_{f}}^{-}$, with $\tau_{k_{f}}^{+} \sim \tau_{k_{f}}^{-} \sim L_{\perp} / \delta v=1.11 \times 10^{5} \mathrm{~s}$. On the other hand, $t_{\mathrm{adv}}=(1 \mathrm{AU}) / U=4.26 \times 10^{5} \mathrm{~s}$, which marginally satisfies the condition $t_{\mathrm{adv}} \gg \tau_{k_{f}}^{+}$. However, if $w_{k_{f}}^{+} / w_{k_{f}}^{-}$were instead $\sim 3$ at $1 \mathrm{AU}$, as in the results of Bavassano et al (2000), then $\tau_{k_{f}}^{+}$would be somewhat larger than $t_{\mathrm{adv}}$. Thus, the inhomogeneity of the solar wind may influence the effects of cross helicity on the spectral indices. However, more detailed modeling of inhomogeneous solar-wind turbulence is beyond the scope of this paper.

\section{Comparison to Other Studies}

In this section, the results of this paper are compared to two recent studies of strong anisotropic incompressible MHD turbulence with cross helicity.

\subsection{Lithwick, Goldreich, \& Sridhar (2007)}

The model for the cascade of energy to larger $k_{\|}$used in this paper is based on the results of Lithwick, Goldreich, \& Sridhar (2007) (hereafter LGS07). As a result, in both studies, if $w^{+}$and $w^{-}$have comparable correlation lengths in the direction of $B_{0}$ at the outer scale, then $\bar{k}_{\|}^{+} \simeq \bar{k}_{\|}^{-}$at all smaller scales. The principal difference between this paper and LGS07 lies in our treatment of the cascade time for the dominant fluctuation type, $w^{+}$. LGS07 argue that if $w_{k_{\perp}}^{+} \gg w_{k_{\perp}}^{-}, \chi_{k_{\perp}}^{+} \sim 1$, and $\chi_{k_{\perp}}^{-} \ll 1$, then the shearing applied by $w^{-}$wave packets on a $w^{+}$wave packet at perpendicular

scale $k_{\perp}^{-1}$ is coherent over a time $\left(k_{\perp} w_{k_{\perp}}^{-}\right)^{-1}$, which greatly exceeds the time $\left(\bar{k}_{\|}^{-} v_{A}\right)^{-1}$ required for a $w^{+}$and $w^{-}$wave packet at perpendicular scale $k_{\perp}^{-1}$ to pass through each other. In contrast, in this paper, it is argued that the coherence time for the straining of the $w^{+}$wave packet is of order the "crossing time" $\left(\bar{k}_{\|}^{-} v_{A}\right)^{-1}$. As a result, the results obtained in this paper for the inertialrange power spectra, degree of anisotropy, cascade time, and energy fluxes are different from those of LGS07.

The approach taken in this paper is motivated by the following argument. As argued by LGS07 and Maron \& Goldreich (2001), the $w^{-}$fluctuations propagate approximately along the 
hypothetical magnetic field lines obtained from the sum of $B_{0}$ and the magnetic field of the $w^{+}$ fluctuations. Let us call these hypothetical magnetic field lines the " $w^{+}$field lines," and let us consider a $w^{+}$wave packet of perpendicular scale $k_{\perp}^{-1}$ and parallel scale $\left(\bar{k}_{\|}\right)^{-1}$, where $\bar{k}_{\|}=\bar{k}_{\|}^{+} \simeq$ $\bar{k}_{\|}^{-}$. Let us work in a frame of reference that moves at speed $v_{A}$ in the $-z$ direction along with the $w^{+}$ fluctuations. Let us also take an initial snapshot of the turbulence at $t=0$ and trace out all of the " $w^{+}$field lines" that pass through our wave packet. The volume filled by these $w^{+}$field lines is the "source region" from which the $w^{-}$wavepackets encountered by our $w^{+}$wave packet originate. If we wait one crossing time $\left(\bar{k}_{\|} v_{A}\right)^{-1}$ and take a new snapshot of the turbulence, then at any given location the $w^{+}$fluctuations will not have changed very much, since $w_{k_{\perp}}^{-} \ll w_{k_{\perp}}^{+}$. However, if we trace out the new $w^{+}$field lines passing through our wave packet, the volume that is filled by these new $w^{+}$field lines will differ substantially from the initial source region at distances $\gtrsim\left(\bar{k}_{\|}\right)^{-1}$ from our wave packet due to the rapid divergence of neighboring field lines in MHD turbulence. In other words, small local changes in $w^{+}$lead to large changes in the connectivity of the $w^{+}$field lines.

To see this, let the $w^{+}$field line that passes through some point $P$ in our wave packet at $t=0$ be called "field line $A$." Let the $w^{+}$field line that passes through point $P$ at $t=\left(\bar{k}_{\|} v_{A}\right)^{-1}$ be called "field line $B$." As before, let us work in a frame of reference that moves at speed $v_{A}$ in the $-z$ direction. Field lines $A$ and $B$ are fixed curves, since they are traced out within two snapshots of the turbulence. If we follow field line $B$ for a distance $\ll\left(\bar{k}_{\|}\right)^{-1}$, it will separate from field line $A$ by some small distance $x$ that is $\ll k_{\perp}^{-1}$. If we continue to follow field line $B$, its separation from field line $A$ is analogous to the separation of two neighboring field lines within a single snapshot of the turbulence. As shown by Narayan \& Medvedev (2001), Chandran \& Maron (2004), and Maron, Chandran, \& Blackman (2004), if a pair of field lines is separated by a distance $x \ll k_{\perp}^{-1}$ at one location, then the distance the field-line pair must be followed before it separates by a distance $k_{\perp}^{-1}$ is a few times the parallel size of an eddy of perpendicular size $k_{\perp}^{-1}$ - i.e., a few times $\left(\bar{k}_{\|}\right)^{-1}$. It turns out that the particular value of $x$ has little effect unless one considers the (irrelevant) case in which $x / d \sim e^{-N}$, where $N$ is large and $d$ is the perpendicular dissipation scale. (Chandran \& Maron 2004) This is because within the inertial range the amount of magnetic shear increases towards small scales; therefore, if $x$ is made very small, then the distance one has to follow the field-line pair in order for $x$ to double becomes very small. Thus, as a result of the rapid divergence of neighboring field lines in MHD turbulence, the "source region" of our $w^{+}$wave packet at $t=\left(\bar{k}_{\|} v_{A}\right)^{-1}$ differs substantially from the source region at $t=0$ at distances $\gtrsim\left(\bar{k}_{\|}\right)^{-1}$ from our wave packet. Because the $w^{-}$fluctuations vary rapidly in the direction perpendicular to the magnetic field, the $w^{-}$wave packets encountered by our $w^{+}$wave packet will decorrelate on a time scale of order the crossing time $\left(\bar{k}_{\|} v_{A}\right)^{-1}$ due to the time evolution of the source region.

It should noted that there are two unexplained aspects of LGS07's model, as pointed out by Beresnyak \& Lazarian (2007). The first concerns the nature of the transition from the weak- 
turbulence regime $\left(\chi_{k_{\perp}}^{+} \ll 1\right.$ and $\left.\chi_{k_{\perp}}^{-} \ll 1\right)$ to the strong-turbulence regime $\left(\chi_{k_{\perp}}^{+} \sim 1\right.$ and $\left.\chi_{k_{\perp}}^{-} \ll 1\right)$. In LGS07's analysis, as one passes from the weak regime to the strong regime, the coherence time for the straining of the $w^{+}$wave packets by $w^{-}$wave packets increases by a factor of $\left(\chi_{k_{\perp}}^{-}\right)^{-1}$ and the energy cascade time $\tau_{k_{\perp}}^{+}$decreases by a factor of $\chi_{k_{\perp}}^{-}$. It is not clear why these large changes should occur across the transition scale. The second issue is that LGS07 find that $E^{+}\left(k_{\perp}\right) \propto k_{\perp}^{-5 / 3}$ and $E^{-}\left(k_{\perp}\right) \propto k_{\perp}^{-5 / 3}$ regardless of the fractional cross helicity. Since the ratio $E^{+}\left(k_{\perp}\right) / E^{-}\left(k_{\perp}\right)$ is independent of wavenumber, it is not clear how pinning could occur in their model, or how the spectra would behave near the dissipation scale.

\subsection{Beresnyak \& Lazarian (2007)}

Beresnyak \& Lazarian (2007) (hereafter BL07) have published an online article on strong MHD turbulence with cross helicity. The following discussion refers to the version of their article that is available electronically as of the writing of this paper. The work of BL07 is similar to this paper in that both studies take the dominant fluctuation type, $w^{+}$, to undergo a weak cascade. Also, equations (31) through (33) of this paper are equivalent to their equation (5), except for the fact that they take the parallel correlation lengths of $w^{+}$and $w^{-}$to differ by a constant multiplicative factor when a power-law solution for the spectra is assumed. (The possibility of more general solutions is claimed by BL07.) On the other hand, there are a number of significant differences between this paper and Beresnyak \& Lazarian's (2007) work. They argue that for the $w^{+}$fluctuations, the dominant nonlinear interactions are between fluctuations with comparable parallel correlation lengths and different perpendicular correlation lengths, whereas for the $w^{-}$fluctuations the dominant interactions are between fluctuations with comparable perpendicular scales. Here, it is argued that for both $w^{+}$and $w^{-}$the dominant interactions are between fluctuations with similar perpendicular scales. When $w_{k_{\perp}}^{+} \gg w_{k_{\perp}}^{-}$and $\chi_{k_{\perp}}^{+} \sim 1$, their Figure 1 suggests that the parallel correlation length of $w^{+}$fluctuations can be less than the parallel correlation length of $w^{-}$fluctuations. It is argued in section 7 of this paper that this can not be the case, because the $w^{+}$fluctuations will imprint their parallel correlation length onto the $w^{-}$fluctuations. They argue that the scalings given by equations (31) and (33) [equivalently, their equation (5)] can not apply if the parallel correlation lengths of the $w^{+}$and $w^{-}$fluctuations are equal, arguing that this would require $\varepsilon^{+}=\varepsilon^{-}$, whereas in this paper the ratio $\varepsilon^{+} / \varepsilon^{-}$depends upon the slopes of the power spectra, as in weak turbulence. They argue that if the $w^{+}$and $w^{-}$fluctuations are driven with the same parallel correlation length at the outer scale, there will be a non-power law part of the solution at large scales that will transition at smaller scales to a power law solution with $w_{k_{\perp}}^{+}$and $w_{k_{\perp}}^{-}$both $\propto k_{\perp}^{-1 / 3}$ and $\bar{k}_{\|}^{ \pm} \propto k_{\perp}^{2 / 3}$. In contrast, in this paper a power-law solution starting at the outer scale is obtained with different scalings for $w_{k_{\perp}}^{+}$and $w_{k_{\perp}}^{-}$, and with $\bar{k}_{\|}^{ \pm}$growing more slowly than $k_{\perp}^{2 / 3}$. 


\section{Conclusion}

This paper proposes a new phenomenology for strong, anisotropic, incompressible MHD turbulence with cross helicity and introduces a nonlinear advection-diffusion equation [equation (15)] to describe the time evolution of the anisotropic power spectra of the $w^{+}$and $w^{-}$fluctuations. It is found that in steady state the one-dimensional power spectra of the energetically dominant $w^{+}$ fluctuations, $E^{+}\left(k_{\perp}\right)$, is steeper than $k_{\perp}^{-5 / 3}$, and that $E^{+}\left(k_{\perp}\right)$ becomes increasingly steep as the fractional cross helicity $\sigma_{c}$ increases. Increasing $\sigma_{c}$ also increases the energy cascade time of the $w^{+}$fluctuations, reduces the turbulent heating power for a fixed fluctuation energy, and increases the anisotropy of the fluctuations at small scales.

Although most of the discussion has focused on forced, steady-state turbulence, the results of this paper can also be applied to decaying turbulence. For example, equations (12) and (13) can be used to estimate the time scale for turbulence to decay. The resulting prediction is that if the

fluctuations are initially excited with $w_{k_{f}}^{+} \gg w_{k_{f}}^{-}$and with comparable parallel correlation lengths at the outer scale, then the turbulence will decay into a state in which $w^{-}$fluctuations are absent, as in the earlier work of Dobrowolny, Mangeney, \& Veltri (1980), Grappin et al (1983), and Lithwick \& Goldreich (2003). This "maximally aligned" state will then be free from nonlinear interactions, and will persist for long times until it damps via linear dissipation.

I thank Chuck Smith, Sebastien Galtier, Bernie Vasquez, Yoram Lithwick, Alex Lazarian, and Andrey Beresnyak for helpful comments and suggestions. This work was supported in part by the NSF/DOE Partnership in Basic Plasma Science and Engineering under grant number AST0613622, by NASA under grant numbers NNX07AP65G and NNH06ZDA001N-SHP06-0071, and by DOE under grant number DE-FG02-07-ER46372.

\section{REFERENCES}

Antonucci, E., Dodero, M. A., \& Giordano, S. 2000, Sol. Phys., 197, 115

Barnes, A. 1966, Phys. Fluids, 9, 1483

Bavassano, B., Pietropaolo, E., \& Bruno, R. 2000, J. Geophys. Res., 105, 15959

Beresnyak, A., \& Lazarian, A. 2006, ApJ, 640, L175

Beresnyak, A., \& Lazarian, A. 2007, arXiv:0709.0554v 1

Bhattacharjee, A. \& Ng, C. S. 2001, ApJ 548, 318

Bieber, J., Matthaeus, W., Smith, C., Wanner, W., Kallenrode, M., \& Wibberenz, G. 1994, ApJ, 420,294 
Biskamp, D., Schwarz, E., \& Drake, J. F. 1996, Phys. Rev. Lett., 76, 1264

Biskamp, D., Schwarz, E., Zeiler, A., Celani, A., \& Drake, J. F. 1999, Phys. Plasmas, 6, 751

Boldyrev, S., Nordlund, A., \& Padoan, P. 2002, Phys. Rev. Lett., 89, 031102

Boldyrev, S. 2005, ApJ, 626, L37

Boldyrev, S. 2006, Phys. Rev. Lett., 96115002

Brodin, G., Stenflo, L., \& Shukla, P. K. 2006, Solar Phys., 236, 285

Bruno, R., \& Carbone, V. 2005, Living Rev. Solar Phys., 2, 4 [Online article: cited Nov. 14, 2007, http://www.livingreviews.org/lrsp-2005-4

Chandran, B. 2004, Sp. Sci. Rev., 292, 17

Chandran, B. 2005, Phys. Rev. Lett., 95, 265004

Cho, J., \& Vishniac, E. 2000, ApJ, 539, 273

Cho, J., Lazarian, A., \& Vishniac, E. 2002, ApJ, 564, 291

Cho, J., \& Lazarian, A. 2002, Phys. Rev. Lett., 88, 245001

Cho, J., \& Lazarian, A. 2003, MNRAS, 345, 325

Cho, J., \& Lazarian, A. 2004, ApJL, 615, L41

Cranmer, S. R. \& van Ballegooijen, A. A. 2005, ApJS, 156, 265

Cranmer, S. R. \& van Ballegooijen, A. A. 2007, ApJS, 171, 520

Dmitruk, P., Milano, L. J., \& Matthaeus, W. H. 2001, ApJ, 548, 482

Dmitruk, P., Matthaeus, W. H., Milano, L. J., Oughton, S., Zank, G. P., \& Mullan, D. J. 2002, ApJ, 575,571

Dobrowolny, M., Mangeney, A., Veltri, P. L. 1980, Phys. Rev. Lett., 35, 144

Elmegreen, B. G., \& Scalo, J., ARAA, 42, 211

Galtier, S., Nazarenko, S. V., Newell, A. C., \& Pouquet, A. 2000, J. Plasma Phys., 63, 447

Galtier, S., \& Chandran, B. 2006, Phys. Plasmas, 3, 114505

Galtier, S., \& Buchlin, E. 2007, ApJ, 656, 560

Goldreich, P., \& Sridhar, S. 1995, ApJ, 438, 763

Goldreich, P., \& Sridhar, S. 1997, ApJ, 485, 680

Goldstein, B. E., Smith, E. J., Balogh, A., Horbury, T. S., Goldstein, M. L., \& Roberts, D. A. 1995, Geophys. Res. Lett., 22, 3393

Grappin, R., Pouquet, A., \& Léorat, J. 1983, A\&A, 126, 51 
Haugen, N. E. L., Brandenburg, A., \& Dobler, W., Phys. Rev. E. 2004, 70, 016308

Higdon, J. 1984, ApJ, 285, 109

Howes, G. G., Cowley, S. C., Dorland, W., Hammett, G. W., Quataert, E., \& Schekochihin, A. A. 2006, ApJ, 651 590-614

Howes, G. G., Cowley, S. C., Dorland, W., Hammett, G. W., Quataert, E., \& Schekochihin, A. A. 2007a, arXiv:0707.3147

Howes, G. G., Dorland, W., Cowley, S. C., Hammett, G. W., Quataert, E., Schekochihin, A. A., \& Tatsuno, T. 2007b, arXiv:0711.4355

Iroshnikov, P. 1963, Astron. Zh. 40, 742

Klein, L. W., Matthaeus, W. H., Roberts, D. A., \& Goldstein, M. L. 1992, Solar Wind Seven; Proceedings of the 3rd COSPAR Colloquium, Goslar, Germany, pp. 197-200.

Kohl, J. L. et al 1998, ApJL, 501, 127

Kolmogorov, A. N. 1941, Dokl. Akad. Nauk SSSR, 30, 299

Kraichnan, R. H. 1965, Phys. Fluids 8, 1385

Kuznetsov, E. A. 2001, J. Exp. Theor. Phys., 93, 1052

Leith, C. E., \& Kraichnan, R. H. 1972, J. Atmos. Sci., 29, 1041

Lithwick, Y., \& Goldreich, P. 2001, ApJ, 562, 279

Lithwick, Y., \& Goldreich, P. 2003, ApJ, 582, 1220

Lithwick, Y., Goldreich, P., \& Sridhar, S. 2007, ApJ, 655, 269 (LGS07)

Luo, Q., \& Melrose, D. 2006, MNRAS, 368, 1151

Maron, J., Chandran, B., \& Blackman, E. 2004, Phys. Rev. Lett., 92, 045001

Maron, J., \& Goldreich, P. 2001, ApJ, 554, 1175

Marsch, E., \& Tu C.-Y. 1996, J. Geophys. Res., 101, 11149

Mason, J., Cattaneo, F., \& Boldyrev, S. 2006, Phys. Rev. Lett., 97, 255002

Matthaeus, W. H., \& Goldstein, M. L. 1982, J. Geophys. Res., 87, 6011

Matthaeus, W. H., \& Goldstein, M. L. 1986, Phys. Rev. Lett., 57, 495

Matthaeus, W. H., \& Montgomery, D. 1980, New York Acad. Sci., 357, 203

Matthaeus, W. H., Zank, G. P., Leamon, R. J., Smith, C. W., Mullan D. J., \& Oughton, S. 1999, Sp. Sci. Rev., 87, 269

Matthaeus, W. H., Mullan D. J., Dmitruk, P., Milano, L., \& Oughton, S. 2002, Non. Proc. Geophys., 9,1 
Matthaeus, W. H., Dmitruk, P., Smith, D., Ghosh, S., \& Oughton, S. 2003, Geophys. Res. Lett., 30, 4-1

Matthaeus, W. H., Pouquet, A., Mininni, P. D., Dmitruk, P., \& Breech, B. 2007, arXiv:0708.0801

Mininni, P., \& Pouquet, A. 2007, Phys. Rev. Lett., 99, 254502

Moffatt, H. K. 1978, Magnetic field generation in electrically conducting fluids (Cambridge, England: Cambridge University Press, 1978)

Montgomery, D., \& Matthaeus, W. 1995, ApJ, 447, 706

Montgomery, D., \& Turner, L. 1981, Phys. Fluids, 24, 825

Müller, W. C., \& Biskamp, D. 2000, Phys. Rev. Lett., 84, 475

Müller, W. C., Biskamp, D., \& Grappin, R. 2003, Phys. Rev. E, 67, 066302

Müller, W. C., \& Grappin, R. 2005, Phys. Rev. Lett., 95, 114502

Ng, C. S., \& Bhattacharjee, A. 1996, ApJ, 465, 845

Ng, C. S., \& Bhattacharjee, A. 1997, Phys. Plasmas, 4, 605

Oughton, S., Matthaeus, W. H., \& Ghosh, S. 1995, LNP, Vol. 462: Small-Scale Structures in Three-Dimensional Hydrodynamic and Magnetohydrodynamic Turbulence, p. 273

Oughton, S., Dmitruk, P., \& Matthaeus, W. H. 2006, Phys. Plasmas, 13, 2306

Padoan, P., Jimenez, R., Nordlund, A., \& Boldyrev, S. 2004, Phys. Rev. Lett., 92, 191102

Perez, J. C., \& Boldyrev, S. 2008, ApJL, 672, L61

Podesta, J. J., Roberts, D. A., \& Goldstein, M. L. 2007, ApJ, 664, 543

Pouquet, A., Sulem, P. L., \& Meneguzzi, M. 1988, Phys. Fluids, 31, 2635

Roberts, D. A., Goldstein, M. L., Klein, L. W., \& Matthaeus, W. H. 1987, J. Geophys. Res., 92, 12023

Schekochihin, A. A., Cowley, S. C., Dorland, W., Hammett, G. W., Howes, G. G., Quataert, E., \& Tatsuno, T 2007b, arXiv:0704.0044

Shebalin, J., Matthaeus, W., \& Montgomery, D. 1983, J. Plasma Phys., 29, 525

Shukla, P. K., Brodin, G., \& Stenflo, L. 2006, Phys. Lett. A, 353, 73

Skilling, J., McIvor, I., \& Holmes, J. 1974, MNRAS, 167, 87P

Smith, C. W. 2003, Solar Wind Ten: Proceedings of the Tenth International Solar Wind Conference, AIP Conference Proceedings, 679, 413

Smith, C. W., Hamilton, K., Vasquez, B., \& Leamon, R. 2006, ApJL, 645, 85

Stone, J. M., Ostriker, E. C., \& Gammie, C. F. 1998, ApJL, 508, 99 
Taylor, G. I. 1938, Proc. R. Soc. London A, 164, 476

Velli, M. 1993, A\&A, 270, 304

Verdini, A., \& Velli, M. 2007, ApJ, 662, 669

Zhou, Y., \& Matthaeus, W. H. 1990, J. Geophys. Res., 95, 10291 